ILC-NOTE-2015-067

DESY 15-094

KEK Preprint 2015-16

LAL $15-188$

MPP-2015-120

SLAC-PUB-16302

June, 2015

\title{
Physics Case for the International Linear Collider
}

\author{
LCC Physics Working Group
}

\begin{abstract}
Keisuke Fuji ${ }^{1}$, Christophe Grojean ${ }^{2,3}$ Michael E. Peskin ${ }^{4}$ (conveners); Tim Barklow ${ }^{4}$, Yuanning GaO ${ }^{5}$, Shinya Kanemura ${ }^{6}$, Hyungdo Kim ${ }^{7}$, Jenny List ${ }^{2}$, Mihoko Nojiri ${ }^{1}$, Maxim Perelstein ${ }^{8}$, Roman Pöschl ${ }^{9}$, Jürgen Reuter ${ }^{2}$, Frank Simon ${ }^{10}$, Tomohiko Tanabe ${ }^{11}$, Jaehoon Yu ${ }^{12}$ James D. Wells ${ }^{13}$; Hitoshi Murayama ${ }^{14,15,16}$, Hitoshi Yamamoto ${ }^{17}$
\end{abstract}

\begin{abstract}
We summarize the physics case for the International Linear Collider (ILC). We review the key motivations for the ILC presented in the literature, updating the projected measurement uncertainties for the ILC experiments in accord with the expected schedule of operation of the accelerator and the results of the most recent simulation studies.
\end{abstract}


${ }^{1}$ High Energy Accelerator Research Organization (KEK), Tsukuba, Ibaraki, JAPAN

2 DESY, Notkestrasse 85, 22607 Hamburg, GERMANY

${ }^{3}$ ICREA at IFAE, Univesitat Autónoma de Barcelona, E-08193 Bellaterra, SPAIN

${ }^{4}$ SLAC, Stanford University, Menlo Park, CA 94025, USA

${ }^{5}$ Center for High Energy Physics, Tsinghua University, Beijing, CHINA

${ }^{6}$ Department of Physics, University of Toyama, Toyama 930-8555, JAPAN

${ }^{7}$ Department of Physics and Astronomy, Seoul National University, Seoul 151-747, KOREA

${ }^{8}$ Laboratory for Elementary Particle Physics, Cornell University, Ithaca, NY 14853, USA

${ }^{9}$ LAL, Centre Scientifique d'Orsay, Université Paris-Sud, F-91898 Orsay CEDEX, FRANCE

${ }^{10}$ Max-Planck-Institut für Physik, Föhringer Ring 6, 80805 Munich, GERMANY

${ }^{11}$ ICEPP, University of Tokyo, Hongo, Bunkyo-ku, Tokyo, 113-0033, JAPAN

${ }^{12}$ Department of Physics, University of Texas, Arlington, TX 76019, USA

${ }^{13}$ Michigan Center for Theoretical Physics, University of Michigan, Ann Arbor, MI 48109, USA

${ }^{14}$ Department of Physics, University of California, Berkeley, CA 94720, USA

15 Theoretical Physics Group, Lawrence Berkeley National Laboratory, Berkeley, CA 94720, USA

${ }^{16}$ Kavli Institute for the Physics and Mathematics of the Universe, University of Tokyo, Kashiwa 277-8583, JAPAN

${ }^{17}$ Department of Physics, Tohoku University, Sendai, Miyagi 980-8578, JAPAN 


\section{Contents}

1 Introduction 1

2 Higgs Boson $\quad 3$

2.1 Introduction . . . . . . . . . . . . . . . . . . . . 3

2.2 Higgs boson observation . . . . . . . . . . . . . . . . . . 5

2.3 Higgs boson coupling measurement . . . . . . . . . . . . . . 7

2.4 Higgs boson self-coupling . . . . . . . . . . . . . . . . . . . . . . . 10

3 Top Quark 13

3.1 Top quark at threshold . . . . . . . . . . . . . . . . . . 14

3.2 Top quark weak and electromagnetic couplings . . . . . . . . . . . 16

4 New Particles $\quad 18$

4.1 Hidden dark matter . . . . . . . . . . . . . . . . . . . . . . . . . . . . 19

4.2 Hidden Higgsino . . . . . . . . . . . . . . . . . . . . . . . . . . . . . 21

4.3 Hidden pseudoscalar Higgs bosons . . . . . . . . . . . . . . . . . . . . 22

4.4 Two-fermion processes . . . . . . . . . . . . . . 23

5 Conclusion $\quad 24$

A Appendix: Table of ILC projected uncertainties 26 


\section{Introduction}

We are now looking forward to the establishment of an international collaboration to construct the International Linear Collider (ILC) in Japan. The physics potential of this machine is known to be very impressive. Its capabilities have been documented, most recently, in Volume 2 of the ILC Technical Design Report [1], in a series of reports to the American Physical Society's study of the future of US particle physics (Snowmass 2013) [2,3,4,5], and in a comprehensive review article [6]. This article gives a brief and accessible review of the main points of these documents.

The ILC is, at this time, the only energy frontier accelerator for the post-LHC era that has moved to the engineering stage and has attracted strong attention from a potential host government. Now, as the project moves to a definite site-specific design, the ILC Parameters Joint Working Group of the Linear Collider Collaboration has presented a realistic plan for the operation of the accelerator, including startup, energy stages, and luminosity upgrades [7]. It is appropriate to update the projected capabilities of the ILC in accordance with this plan.

In this paper we present a summary of the ILC physics case and updates of the expected measurement uncertainties, including results from new simulation studies. In the program from the ILC Parameters Joint Working Group [7], the ILC would have an initial phase in which it accumulates $500 \mathrm{fb}^{-1}$ at $500 \mathrm{GeV}$, then $200 \mathrm{fb}^{-1}$ at $350 \mathrm{GeV}$, then $500 \mathrm{fb}^{-1}$ at $250 \mathrm{GeV}$. After a luminosity upgrade, the ILC would accumulate an additional $3500 \mathrm{fb}^{-1}$ at $500 \mathrm{GeV}$, then an additional $1500 \mathrm{fb}^{-1}$ at $250 \mathrm{GeV}$. Results quoted here for the "initial" and "full" ILC data sets are based on this scheme.

The most important aspects of the ILC physics program are: (1) measurement of the properties of the newly-discovered Higgs boson with very high precision; (2) measurement of the properties of the top quark with very high precision; (3) searches for and studies of new particles expected in models of physics at the $\mathrm{TeV}$ energy scale. The specific capabilities of the ILC in these areas are reviewed in the various sections of this report. The physics program of the ILC is still broader, encompassing precision electroweak measurements, detailed studies of the $W$ and $Z$ boson couplings, tests of Quantum Chromodynamics, and other topics. A complete survey is given in Ref. [1].

Before we begin, we should make two general points about the role of the ILC in the current situation in particle physics. The first is that the discovery of the Higgs boson at the CERN Large Hadron Collider [8,9] is a milestone in the history of particle physics that changed our perspective on the goals of this field. We now have in hand the complete particle spectrum of a "Standard Model" that could be correct up to very high energies. It is possible that this theory of particle physics could be correct up to energies thirteen orders of magnitude higher than our current experiments. However, this would be unfortunate, because this model is inadequate 
in several important respects. First, it does not explain the most basic fact about the Higgs field, why it is that this field forms a condensate that fills space and gives rise to the masses of all known elementary particles. Second, it has no place for the particle or particles that make up cosmic dark matter, a neutral, weakly interacting substance that, according to astrophysical observation, makes up $85 \%$ of the mass in the universe and $25 \%$ of its total energy. Third, it does not explain the asymmetry in the amount of matter and antimatter in the universe. One might add to this list many more fundamental questions, for example, why quarks and leptons, which make up observed matter, have the quantum numbers that they do. However, these three questions are the keys to progress through experiment. The most pressing issue in particle physics today is that of where and how the Standard Model breaks down. If the questions just listed have answers given by current theoretical proposals, new particles and forces beyond the Standard Model should appear at the leading accelerators currently operated and planned - the LHC and the ILC.

In the discussion to follow, we will compare the capabilities of the LHC and the ILC. However, it is also important to realize that the experimental programs at these accelerators differ in essential ways. The LHC gives access to high energies for direct production of new particles. However, this comes at a price. The rates of production of proposed new particles are typically $10^{-10}-10^{-12}$ of the proton-proton total cross section. Even after selection of characteristic event types, these processes typically represent only about $10 \%$ of the total yield, over a background consisting of complex Standard Model reactions. This limits both the range of new processes that can be observed and the precision with which rates can be measured.

At the ILC, and more generally in electron-positron collisions, the situation is qualitatively different. The processes that we wish to study are large fractions of the total electron-positron annihilation cross section. Event selections give high purity, over backgrounds that are straightforward to compute. For the study of a heavy particle, all decay modes can be observed, and systematic errors on measured rates are at the $0.1 \%$ level. This is a powerful and unique capability that we can apply to the Higgs boson and top quark - the two known particles most directly connected to the questions we have listed above - and to any new particles that might appear in the energy range that the ILC will study. Precision measurements at the ILC can not only prove the existence of new particles with masses well above the $e^{+} e^{-}$collision energy but also can give detailed information about their properties. We will see examples of this in all three sections below.

The second point is a perspective on the longer-range future of high-energy physics. Our field's need for larger and more powerful accelerators has driven us to be more globalized than any other field of science. Today, there is one high-energy protonproton collider in the world, the LHC. Its construction was made possible by the existing complex of tunnels and infrastructure at CERN. At the moment, a large fraction of the experimental particle physicists in the world are collaborators in the 
two large experiments ATLAS and CMS at the LHC. This insures CERN's current stature as the major international center of particle physics.

For electron-positron collisions, any facility at energies much higher than those already realized must be a linear collider in a long, straight tunnel. The ILC infrastructure will provide a basis for collisions at $500 \mathrm{GeV}$. The technology for ILC can extend the reach of the machine to $1 \mathrm{TeV}$, as envisioned in the ILC TDR [10]. Beyond this, the ILC laboratory will provide a setting in which new generations of technology will provide electron-positron collisions at even higher energies. It will provide a new world laboratory that will be the global host for experiments with electron and positron beams into the long-term future.

With this background, we now review the capabilities promised by the ILC for experiments on the Higgs boson (Section 2), the top quark (Section 3), and proposed new particles (Section 4). An appendix gives a table of the projected measurement errors for the most important parameters. We recommend that these numbers be used in discussions of the ILC physics prospects and in comparisons of the ILC with other proposed facilities.

\section{Higgs Boson}

\subsection{Introduction}

It is a property of the Standard Model of particle physics that, $10^{-10}$ seconds after the Big Bang, empty space-time made a transition to a new state filled with a uniform field. This field, the Higgs field, breaks symmetries of the Standard Model that forbid the masses of quarks, leptons, and vector bosons. Its uniform field value is therefore responsible for the masses of these particles. The observation of the Higgs boson at the LHC gives evidence that this set of ideas is correct. In particular, the measurements of $Z$ boson polarization in $h \rightarrow Z Z^{*} \rightarrow 4$ lepton events already gives strong evidence that the Higgs boson couples to the $Z$ boson in the specific manner required to give the $Z$ boson its mass. More generally, the LHC measurements of the various production and decay reactions of the Higgs boson are all consistent with the picture of the Higgs field as the origin of all elementary particle masses. The current situation is illustrated in Fig. 1(a) [11]. Over the next decade, it is likely that the LHC will provide additional evidence toward this conclusion. However, this evidence only addresses the question of how the weak interaction symmetry is broken. It does not address the question of why the symmetry is broken or why the Higgs field acquires its nonzero value.

With the measurement of the mass of the Higgs boson, now known to $0.2 \%$ accuracy, the parameters of the Standard Model are fixed. All further properties of the 

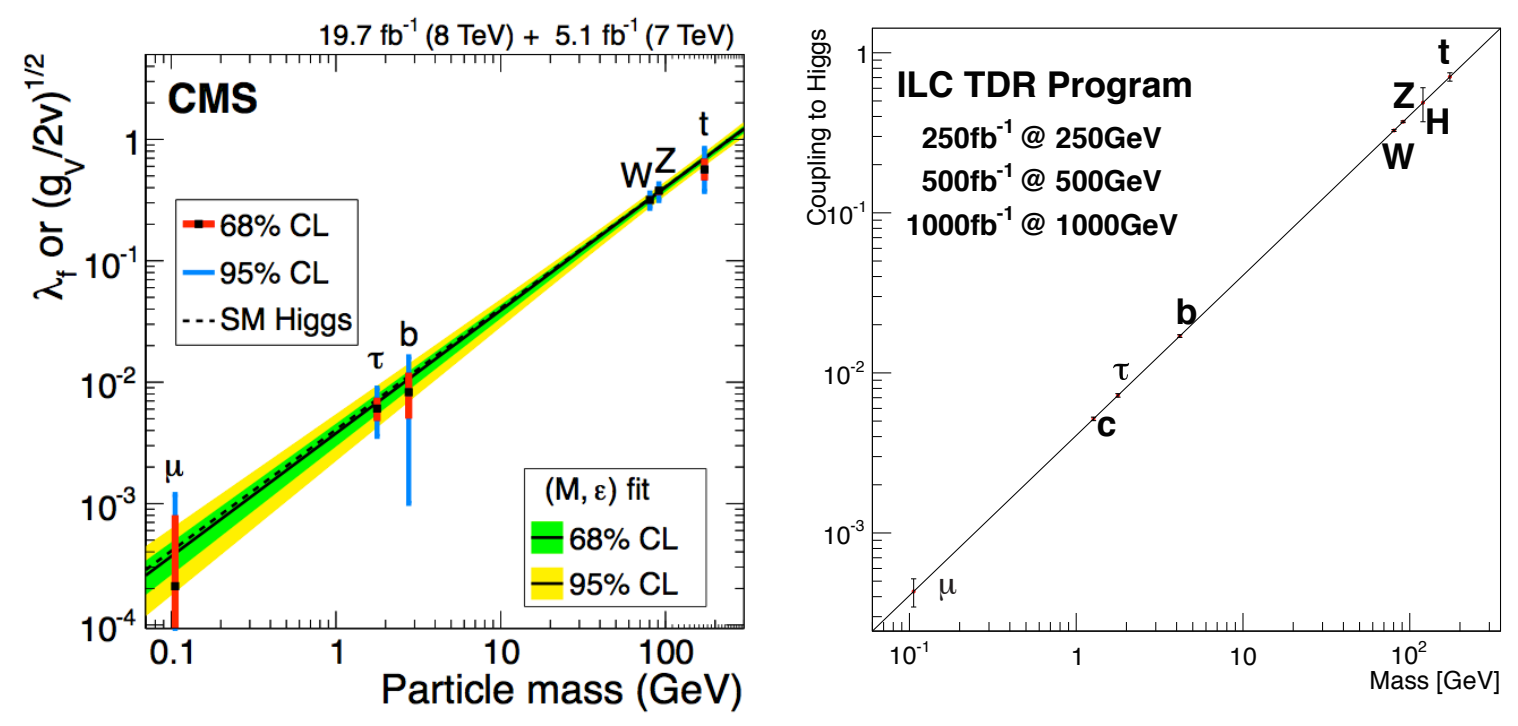

Figure 1: The Standard Model predicts that the Higgs couplings to fundamental fermions are linearly proportional to the fermion masses, whereas the couplings to bosons are proportional to the square of the boson masses. Left: the CMS fit to the current Higgs data, showing consistency with this prediction, from Ref. [11]. Right: the expected improvement in the precision in the measurement of the Higgs couplings at the ILC, from Ref. [1].

Higgs boson are predicted by the model. The observation of any deviation from these predictions would be a clear indication of new physics beyond the Standard Model.

Different models of new physics beyond the Standard Model lead to different patterns of deviation from the predicted Higgs boson couplings. In supersymmetric models, and more generally in models with more than one fundamental Higgs field, the largest deviations are expected to be found in the couplings to the down-type quarks and leptons and in the effective couplings to photons and gluons. In models in which the Higgs boson is composite, the effects of compositeness produce a uniform decrease in all of the Higgs couplings. Such models may also have partial top quark compositeness and heavy top partners; these effects induce further shifts of the Higgs couplings to top quarks and to photons and gluons. Thus, the measurement of the couplings of the Higgs boson will give evidence on the question of whether the Higgs boson is a fundamental scalar particle - the first ever observed - or a composite of more fundamental constituents. Looking for deviations of the Higgs couplings is also a way to probe the naturalness of the weak scale. Indeed, general arguments $[12,13]$ imply that any new physics that screens the Higgs mass from large quantum corrections generically leads to deviations in the Higgs couplings to photons and gluons at least as large as 1\%. Supersymmetric and composite Higgs models are prime examples of this general pattern. 
However, the size of deviations in the Higgs couplings is limited by LHC exclusions of new particles and by precision weak interaction measurements. Taking these constraints into account, the deviations predicted in all of the models above are generically small, at the level of about $5 \%$ or less, varying as $m_{h}^{2} / M^{2}$, where $M$ is the mass of the new particles predicted in the model. The loop-induced couplings of the Higgs boson to $\gamma \gamma$ and $g g$ receive contributions from the Standard Model particles $t$ and $W$, but also, possibly, from new heavy particles. In the fits that we present below, we consider these couplings to be independent of the couplings of $t$ and $W$ to the Higgs boson.

At the LHC, the uncertainties in the Standard Model predictions for the rates of Higgs processes are also of the order of $5 \%$, and systematic errors on detection probabilities are of the same order. In addition, only a subset of the Higgs decays can be observed directly. Because not all Higgs decays are observed, there are further ambiguities, discussed below. Thus, the goal for Higgs boson experiments, the measurement of the individual Higgs couplings to accuracies of better than $1 \%$, can be met only by experiments at an electron-positron collider. The improvement expected from the ILC over the current measurements is shown in Fig. 1(b) [1].

\subsection{Higgs boson observation}

As we have discussed above, the ILC will study the Higgs boson using the features available at an $e^{+} e^{-}$collider: a well-defined initial state, absence of strong-interaction backgrounds, and controlled and calculable backgrounds from electroweak processes. The relatively quiet environment of $e^{+} e^{-}$collisions also allows the construction of detectors with higher intrinsic precision and heavy-flavor tagging efficiency than is possible at the LHC. These detectors essentially reconstruct all events in terms of fundamental particles such as leptons, quarks, and gauge bosons. There are three major Higgs boson production processes at the ILC: $e^{+} e^{-} \rightarrow Z h$ ("higgsstrahlung"), $e^{+} e^{-} \rightarrow \nu_{e} \bar{\nu}_{e} h$ ("W fusion"), and $e^{+} e^{-} \rightarrow e^{+} e^{-} h$ (" $Z$ fusion"). For each of these, we will be able to separately identify all of the major Higgs decay modes, such as $h \rightarrow b \bar{b}$, $W W^{*}, c \bar{c}, \tau \tau$, and $g g$, with high efficiency. It is worth recalling that the decays of the Higgs boson to quarks are very difficult to observe at the LHC. The decay $h \rightarrow b \bar{b}$ can be observed only in special kinematics, and it seems extremely challenging to observe $h \rightarrow c \bar{c}$ or $h \rightarrow g g$ (though the latter coupling can be probed in Higgs production). On the other hand, if one anticipates a special role for the top quark in electroweak symmetry breaking, it would be important to measure the Higgs coupling to $c \bar{c}$ as a reference value to understand any deviations from the Standard Model predictions in the Higgs couplings to $t \bar{t}$ and $g g$.

The control of electron and positron beam polarization that the ILC will make available allows experimenters to select Higgs reactions or to change the mixture of signal and background. For example, the $W$ fusion process $e^{+} e^{-} \rightarrow \nu_{e} \bar{\nu}_{e} h$ proceeds 


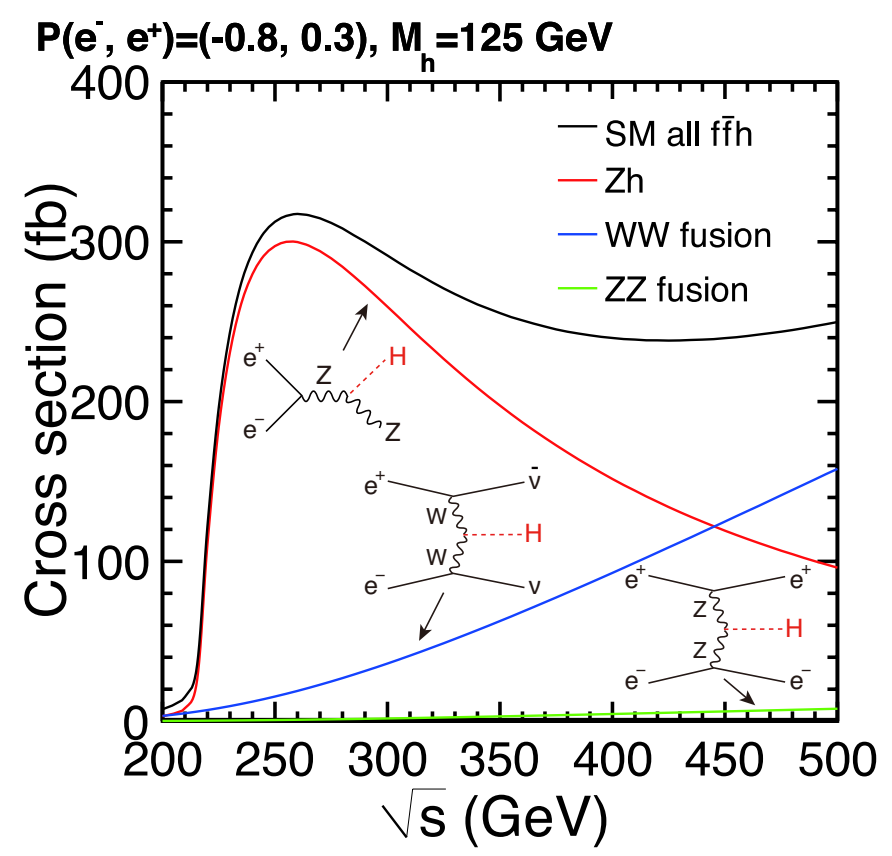

Figure 2: Cross sections for the three major Higgs production processes as a function of center of mass energy, from Ref. [1].

only via collisions of left-handed electrons with right-handed positrons. As a consequence, its cross section can be enhanced by a factor of about 2 with the polarized electron and positron beams available at the ILC. Figure 2 plots the cross sections for the single Higgs boson production at the ILC with the left-handed polarization combinations: $P\left(e^{-}, e^{+}\right)=(-0.8,+0.3)$. The figure tells us that at a center of mass energy of $250 \mathrm{GeV}$ the higgsstrahlung process attains its maximum cross section, providing about 160,000 Higgs events for an integrated luminosity of $500 \mathrm{fb}^{-1}$. At $500 \mathrm{GeV}$, a sample of $500 \mathrm{fb}^{-1}$ gives another 125,000 Higgs events, of which $60 \%$ are from the $W$ fusion process [14]. With these samples of Higgs events, we can measure the rates for Higgs production and decay for all of the major Higgs decay modes.

The higgstrahlung process $e^{+} e^{-} \rightarrow Z h$ offers another special advantage. By identifying the $Z$ boson at a well-defined laboratory energy corresponding to the kinematics of recoil against the $125 \mathrm{GeV}$ Higgs boson, it is possible to identify a Higgs event without looking at the Higgs decay at all. This has three important consequences. First, as we will describe below, it gives us a way to determine the total width of the Higgs boson and the absolute normalization of the Higgs couplings. Second, it allows us to observe Higgs decays to invisible or exotic modes. Decays of the Higgs boson to dark matter, or to other long-lived particles that do not couple to the Standard Model interactions, can be detected down to branching ratios below $1 \%$. 


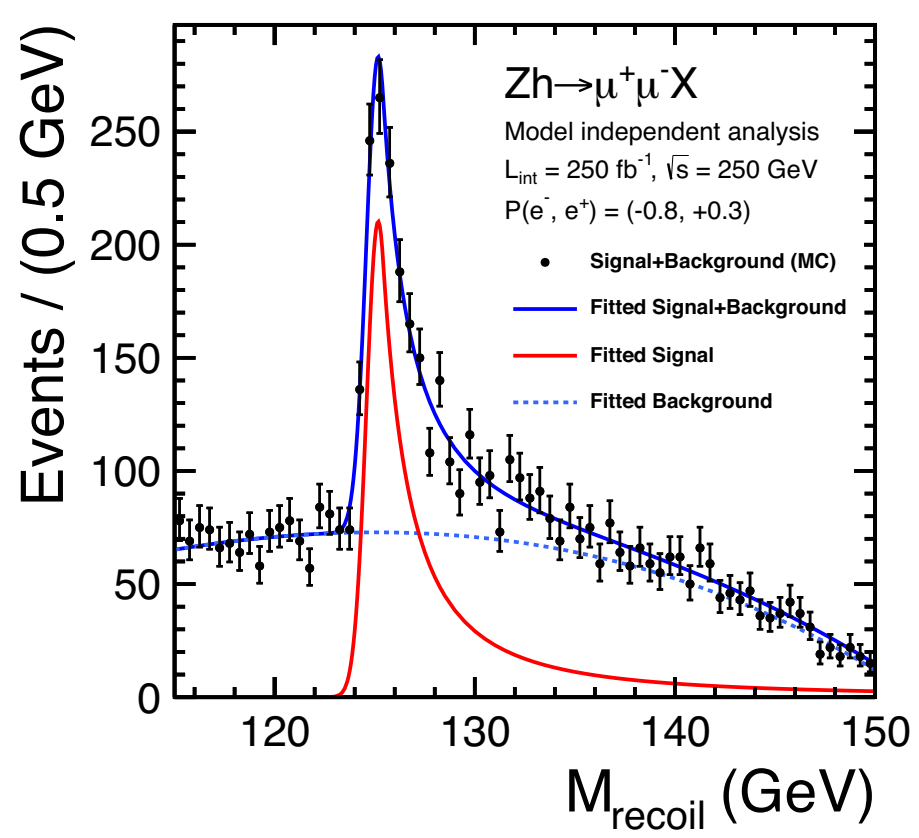

Figure 3: Recoil mass distribution for the process: $e^{+} e^{-} \rightarrow Z h$ followed by $Z \rightarrow \mu^{+} \mu^{-}$ decay for $m_{h}=125 \mathrm{GeV}$ with $250 \mathrm{fb}^{-1}$ at $\sqrt{s}=250 \mathrm{GeV}$, based on Ref. [15].

Finally, measurement of the decay of the $Z$ to $e^{+} e^{-}$or $\mu^{+} \mu^{-}$gives a very precise determination of the mass of the Higgs boson. The mass of a particle recoiling against a lepton pair is given by

$$
M_{X}^{2}=\left(p_{C M}-\left(p_{\ell^{+}}+p_{\ell^{-}}\right)\right)^{2},
$$

where $p_{C M}$ is the 4-momentum of the annihilating electron-positron system. The expected recoil mass distribution for a $m_{h}=125 \mathrm{GeV}$ Higgs boson with $250 \mathrm{fb}^{-1}$ at $\sqrt{s}=250 \mathrm{GeV}$ is shown in Fig. 3. This measurement allows us to determine the Higgs mass to better than $30 \mathrm{MeV}$ and the cross section to a sub- $\%$ level [2]. The precision of the cross section can be further improved by adding events with decay of the $Z$ to quarks.

\subsection{Higgs boson coupling measurement}

To compare Higgs boson rate measurements to the Standard Model expectations, it is important to note that what is actually measured is the rate for a production and decay process. This is proportional to the cross section for Higgs production multiplied by the branching ratio (BR), which is related to the partial width into the observed channel through the familiar formula

$$
B R(h \rightarrow A \bar{A})=\Gamma(h \rightarrow A \bar{A}) / \Gamma_{h},
$$


where $\Gamma_{h}$ is the total rate of Higgs decay or the total width of the Higgs boson as a resonance. In the Standard Model, $\Gamma_{h}$ is very small, too small to be measured directly as a resonance width. Unfortunately, we must determine $\Gamma_{h}$ to learn the absolute sizes of the Higgs boson couplings.

At the LHC, all determinations of $\Gamma_{h}$ to high accuracy require model-dependent assumptions. At the ILC, however, we can use the fact that all Higgs decay modes can be identified in the higgsstrahlung process using the recoiling $Z$ boson to measure certain Higgs couplings directly. The total rate for higgsstralung is proportional to the $Z Z h$ coupling. The rate for the $W$ fusion process

$$
e^{+} e^{-} \rightarrow \nu \bar{\nu} h \quad \text { with } h \rightarrow b \bar{b},
$$

divided by $B R(h \rightarrow b \bar{b})$ determined with higgsstrahlung, gives the $W W h$ coupling. These measurements then determine $\Gamma(h \rightarrow Z Z)$ and $\Gamma(h \rightarrow W W)$. Combining these results with the measured branching ratios using (2), the ILC measurements give a model-independent determination of $\Gamma_{h}$. That result in turn fixes the absolute size of all other Higgs couplings.

The most statistically powerful determination of the Higgs width $\Gamma_{h}$ uses the $W$ fusion process, which turns on at $e^{+} e^{-}$center of mass energies above $250 \mathrm{GeV}$, as shown in Fig. 2. Thus, the most precise coupling measurements from the ILC require data-taking at energies of $350 \mathrm{GeV}$ or above. The coupling precisions can be further improved by increasing the data sample or by running at still higher energies.

Because the decay of the Higgs boson to $\gamma \gamma$ is rare, with a branching ratio of $0.2 \%$ in the Standard Model, it will be difficult for the ILC to gather large statistics for this decay. Fortunately, the $\gamma \gamma$ and $Z Z$ decay modes of the Higgs boson are the modes that are most straightforward for the LHC experiments. The LHC is expected to measure the ratio of branching ratios $B R(h \rightarrow \gamma \gamma) / B R(h \rightarrow Z Z)$ very accurately, using a technique in which the systematic errors largely cancel. In Ref. [16], it is estimated that this ratio of branching ratios can be measured to $2 \%$ accuracy. Combination of this with the $Z Z h$ coupling measurement from the ILC will allow us to reach the required 1\%-level precision also for the $h \rightarrow \gamma \gamma$ coupling [17]. This synergy is illustrated in the $\gamma \gamma$ entries of the summary figures cited below.

Since the top quark is the heaviest particle in the Standard Model and hence most strongly coupled to the Higgs sector, the Higgs boson coupling to the top quark could contain special effects and should be measured independently. This coupling is not directly accessible from the Higgs decay measurements. To determine it, we use the reaction $e^{+} e^{-} \rightarrow t \bar{t} h$ with $h$ observed in its $b \bar{b}$ decay. The rate of this reaction is proportional to $B R(h \rightarrow b \bar{b})$, but that quantity will have been measured very accurately in the program described above. We can then determine the Higgs coupling to top quarks by just counting the number of $t \bar{t} h$ events. Full-simulation studies for ILC at $500 \mathrm{GeV}$ show [1] that this will provide a $6.3 \%$ measurement of the $t \bar{t} h$ coupling 


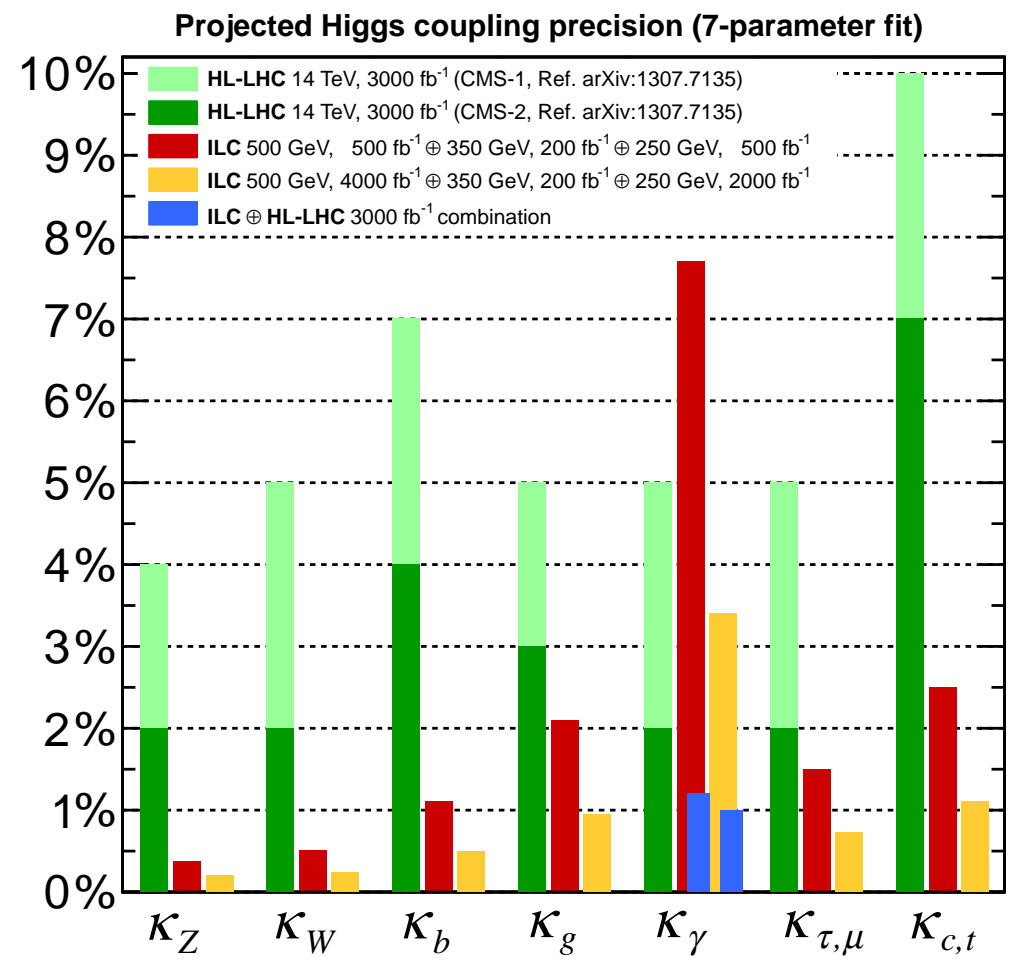

Figure 4: Relative precisions for the various Higgs couplings extracted using the modeldependent fit used in the Snowmass 2013 study [18], applied to expected data from the High-Luminosity LHC and from the ILC. Here, $\kappa_{A}$ is the ratio of the $A \bar{A} h$ coupling to the Standard Model expectation. The red bands show the expected errors from the initial phase of ILC running. The yellow bands show the errors expected from the full data set. The blue bands for $\kappa_{\gamma}$ show the effect of a joint analysis of High-Luminosity LHC and ILC data. 
with the full ILC data set. It is worth pointing out that the energy of $500 \mathrm{GeV}$ is very close to the production threshold for this process. Thus, the full ILC energy of $500 \mathrm{GeV}$ is necessary to achieve these goals. On the other hand, a slight increase of the center of mass energy, by $10 \%$, enhances the cross section by a factor of about four and improves the precision to $3 \%$ for the same integrated luminosity.

In Fig. 4, we compare the uncertainties in Higgs couplings expected from the High-Luminosity LHC and from the two phases in the evolution of the ILC program. Because the LHC experiments cannot measure all Higgs decay modes, they cannot make a model-independent determination of the Higgs width $\Gamma_{h}$. Thus, in this figure, the couplings are determined with constraints that fix the unobserved modes. Following Ref. [18], this fit assumes that the fractional shift in the Higgs couplings is equal for $u, c, t$, for $d, s, b$, and for $e, \mu, \tau$, and that there is no Higgs decay to invisible or exotic particles. The large green bars give the uncertainty projections from the CMS Collaboration assuming current values of systematic errors. The heavier green bars assume that systematic errors can be decreased by the same factor as statistical errors, by a factor of 12 from today to the end of the High-Luminosity LHC program [19] Projections by the ATLAS collaboration are similar [16]. The ILC estimates are based on current full-simulation studies and the ILC program described in Ref. [7].

Figure 5 shows the estimated uncertainties from the ILC for a model-independent fit to the Higgs boson couplings in which all Higgs couplings, including couplings to inivisible and exotic modes, are separately taken as free parameters. We see that, in these model-independent determinations, most couplings reach the required precision of $1 \%$ or better in the course of the ILC program. As noted above, running the ILC at $550 \mathrm{GeV}$ rather than $500 \mathrm{GeV}$ would give precisions of $9 \%$ and $3 \%$ in the two entries for the $t \bar{t} h$ coupling.

Figure 6 shows the power of the ILC precision to distinguish different models of new physics through Higgs boson measurements. The two panels illustrate the shifts in the Higgs couplings from the Standard Model predictions expected in two representative models of new physics. The error intervals are those expected from the full ILC data set using a model-independent analysis. Similar illustrations for additional models of new physics are presented in Ref. [20]. It is important not only to observe deviations from the Standard Model but also to use the observed pattern of deviations as a clue to the correct model that lies behind it. The ILC will give us that capability.

\subsection{Higgs boson self-coupling}

There is one more important Higgs coupling not shown in Fig. 5. This is the trilinear Higgs self-coupling, which determines the shape of the potential energy for 


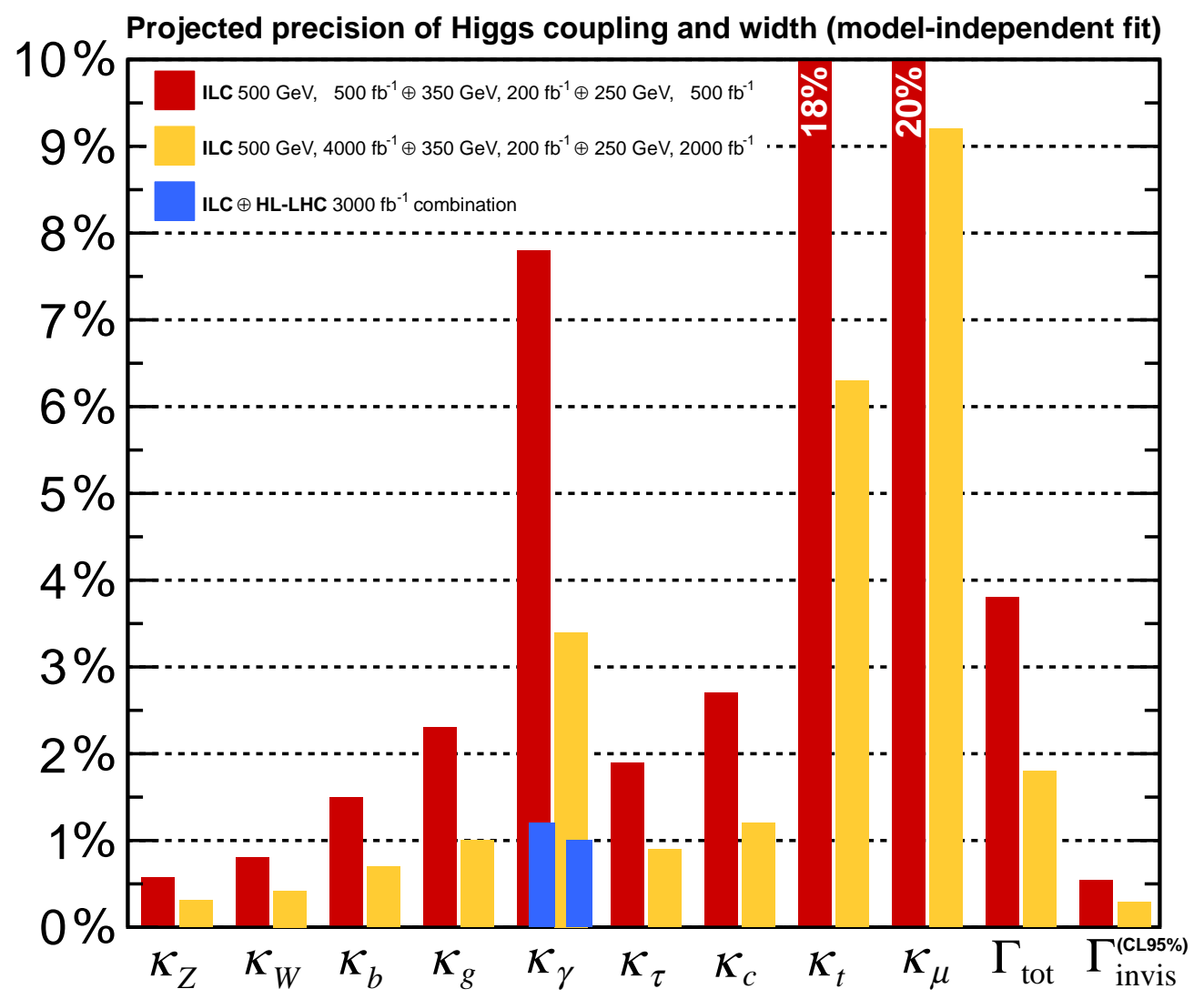

Figure 5: Relative precisions for the various Higgs couplings extracted from a modelindependent fit to expected data from the ILC. The notation is as in Fig. 4. 

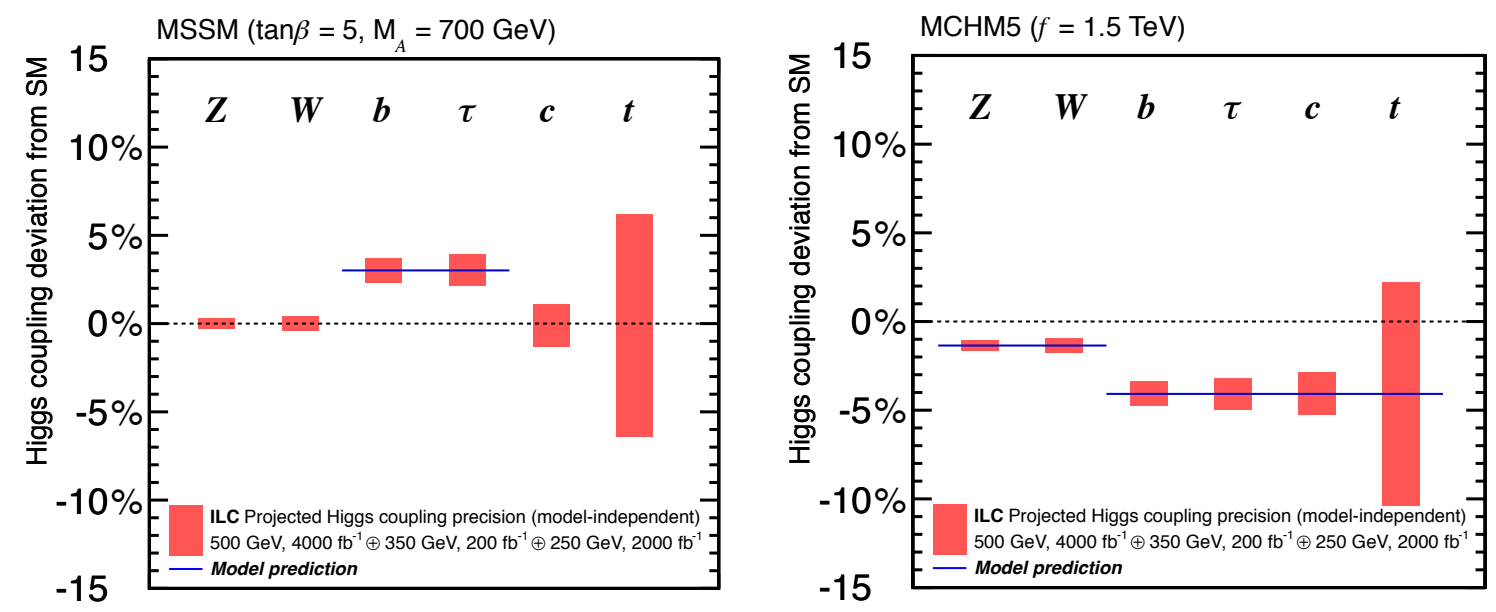

Figure 6: Two examples of models of new physics and their predicted effects on the pattern of Higgs boson couplings. Left: a supersymmetric model. Right: a model with Higgs boson compositeness. The error bars indicate the $1 \sigma$ uncertainties expected from the modelindependent fit to the full ILC data set.

the Higgs field. The value of this coupling gives evidence on the nature of the phase transition in the early universe from the symmetric state of the weak interaction theory to the state of broken symmetry with a nonzero value of the Higgs field.

In the Standard Model, this transition is predicted to be continuous [21]. However, if the transition were first-order, it would put the universe out of thermal equilibrium and, through possible CP violating interactions in the Higgs sector, it would allow the generation of a nonzero baryon-antibaryon asymmetry. This is not the only theory for the baryon-antibaryon asymmetry, but it is the only theory in which all relevant parameters can potentially be measured at accelerators, setting up a quantitative experimental test.

The first step would be to test the nature of the phase transition. Models in which the phase transition is first-order typically require the Higgs self-coupling to differ from the value predicted by the Standard Model [22]. The Higgs self-coupling can be a factor of 2 larger in some models [23].

At the High-Luminosity LHC, double Higgs production can be detected in wellchosen final states, for example, the state in which one Higgs boson decays to $\gamma \gamma$, providing a clean signal, while the other decays to $b \bar{b}$, providing the maximum rate. This process should eventually be observed at the LHC, though current fast-simulation studies are rather pessimistic [24].

At the ILC at $500 \mathrm{GeV}$, pairs of Higgs bosons are produced through $e^{+} e^{-} \rightarrow Z h h$. All Higgs decay modes are observable and will contribute to the measurement. The modes $h h \rightarrow b \bar{b} b \bar{b}$ and $h h \rightarrow b \bar{b} W W$ have been studied in full simulation at the center 
of mass energy $500 \mathrm{GeV}$. Combining the preliminary results of these two channels only, these ILC simulations currently predict a precision of $27 \%$ on the Higgs selfcoupling with the full ILC data set. This would already be more than $3 \sigma$ evidence for the existence of the Higgs self-coupling at the Standard Model value. It gives a substantial discovery potential for models of the Higgs phase transition that predict a larger value. Further improvements from the inclusion of more decay modes and refined analyses are under study.

Running at higher energy allows one to study the process $e^{+} e^{-} \rightarrow \nu \bar{\nu} h h$, whose cross section increases with energy and has a different functional dependence on the self-coupling from the $Z h h$ reaction. The decay mode $h h \rightarrow b b b b$ has been studied in full simulation at $1 \mathrm{TeV}$. Using both the $h h \rightarrow b b b b$ and $h h \rightarrow b b W W$ modes at $1 \mathrm{TeV}$, we expect a precision on the Higgs boson self-coupling of $16 \%$ for $2000 \mathrm{fb}^{-1}$ and $10 \%$ for $5000 \mathrm{fb}^{-1}[25,26]$.

\section{Top Quark}

Among the six quarks of the Standard Model of particle physics, the top quark has a special role. It is the heaviest of the six and, we now know from the LHC, the heaviest particle with the quantum numbers of a Standard Model quark. By virtue of its large mass, the top quark has the strongest coupling of any known particle to the Higgs field or fields that generate the spontaneous breaking of the weak interaction symmetry.

The top quark has a central position in all models of new physics beyond the Standard Model. Such models must contain new particles that are, in a well-defined sense, partners of the top quark. In the most important models, including supersymmetry and models with new space-time dimensions, it is the coupling of the Higgs fields to the top quark and its partners that causes the Higgs field to develop a symmetry-breaking value in all of space.

Through particle physics experiments, we have a very detailed knowledge of the other heavy quarks, $c$ and $b$. The information about these quarks comes from both hadron and from $e^{+} e^{-}$colliders. In general, hadron colliders supply information on rare processes and on the quark-gluon coupling, while $e^{+} e^{-}$colliders supply information on the weak and electromagnetic couplings. Hadron colliders require specific, relatively simple, decays to recognize heavy quarks, while at $e^{+} e^{-}$colliders, heavy quark production provides a large and well characterized part of the total event rate. For this reason, the full, very rich, pattern of weak interaction decays of $c$ and $b$ has been observed mainly at $e^{+} e^{-}$colliders. Though the lifetime of the $b$ quark was measured very accurately at hadron colliders, the pattern of CP-violating couplings of the $b$ quark were first measured at $e^{+} e^{-}$colliders at KEK-B and PEP-II. These 
experiments gave the crucial evidence for the Kobayashi-Maskawa model of CP violation $[27,28]$.

For the top quark, we have today only the hadron collider side of the story. The pair production threshold of the top quark is around $350 \mathrm{GeV}$, higher than the center of mass energy of any $e^{+} e^{-}$collider that has operated so far. The top quark was discovered at the Fermilab Tevatron and has been studied with high statistics at the LHC. However, the difficulties of recognizing and reconstructing top quark events in the presence of large backgrounds, as well as theoretical uncertainties associated with the interpretation of the hadron collider data, limit the accuracy of measurements on this particle. The ILC will allow us, for the first time, to study the top quark in $e^{+} e^{-}$ collisions, where we will be able to access the widest variety of final states with high fidelity.

There are two somewhat distinct physics programs on the top quark at the ILC. The first is the study of the threshold for $t \bar{t}$ production. This is the "hydrogen atom of strong interactions", the first situation in which bound states predicted by QCD can be studied in a setting that is free of the nonperturbative, quark-confining, part of the interaction. ILC measurements near $350 \mathrm{GeV}$ will test this theory and also measure the mass of the top quark to a precision below $10^{-3}$. In addition, other properties such as the total width will be accessible via these measurements. The second is the study of top quark production and decay at $500 \mathrm{GeV}$. These measurements will make use of accurate reconstruction of $t \bar{t}$ events to probe the full structure of the top quark coupling to the electroweak interactions and provide excellent sensitivity to physics beyond the Standard Model.

\subsection{Top quark at threshold}

If the top quark were stable, the $t \bar{t}$ system would show prominent resonances at the $1 \mathrm{~S}, 2 \mathrm{~S}$, etc., quark-antiquark bound states. The QCD potential between $t$ and $\bar{t}$ is expected to be approximately Coulombic. The Bohr radius is expected to be sufficiently small that the nonperturbative, confining part of the potential would have a negligible effect on the properties of the lowest bound states. In reality, the top quark decays, with a width predicted to be about $1.4 \mathrm{GeV}$. However, this width serves only to smear out the resonances in a well-defined way without adding new ambiguities. The theory of the top quark threshold has been worked out to high precision $[29,30,31]$, so very accurate predictions are available to compare to experimental measurements. The predicted threshold shape and simulated ILC measurements are shown in Fig. 7.

The top quark threshold region occupies an interval of less than $10 \mathrm{GeV}$ in the energy of the $t \bar{t}$ system. The study of the shape of the threshold is completely inaccessible to measurements at hadron colliders, due to the poor definition of the parton-parton center of mass energy in hadronic reactions. 


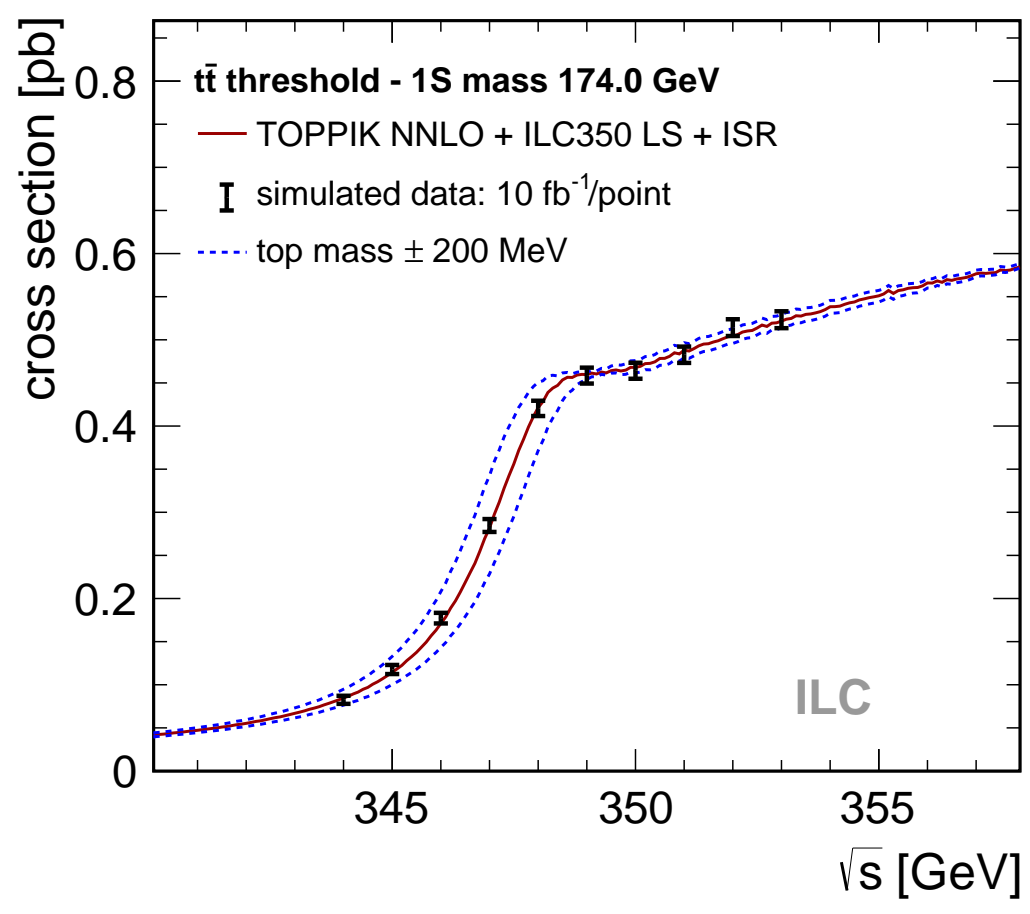

Figure 7: Top quark pair production threshold, including the luminosity spectrum of the ILC, and simulated data points, corresponding in total to one year at design luminosity, from Ref. [33].

The real part of the pole corresponding to the $1 \mathrm{~S}$ bound state is a precisely defined quantity that can be extracted from the threshold measurements. This mass parameter can be determined to about $50 \mathrm{MeV}$ in the ILC program. The accuracy of this measurement is limited by the precision of the theoretical prediction of the threshold shape, now known at $\mathrm{N}^{3} \mathrm{LO}$ [31,32]. For the $200 \mathrm{fb}^{-1}$ data set expected near $350 \mathrm{GeV}$ [7], the expected statistical errors in a 3-parameter fit to the threshold shape are $17 \mathrm{MeV}$ for $m_{t}, 26 \mathrm{MeV}$ for $\Gamma_{t}$, and $4.2 \%$ for the top quark Yukawa coupling [33,34]. Uncertainties from knowledge of the ILC beam parameters are expected to be still smaller.

The 1S top quark mass is connected to other theoretically precise definitions of the top quark mass, such as the $\overline{M S}$ mass, to an accuracy of about $10 \mathrm{MeV}$ [35]. The error in converting an on-shell top quark mass to the $\overline{M S}$ mass is more than an order of magnitude greater. Further, the mass usually quoted from Tevatron and LHC data is simply the input value used in a popular Monte Carlo event generator; its connection to theoretically precise values is not understood. At the High-Luminosity $\mathrm{LHC}$, it is estimated that the $\overline{M S}$ top quark mass can be extracted to an accuracy of 
about $500 \mathrm{MeV}$ in a theoretically precise way by measuring the jet-lepton endpoint in leptonic top decays [36].

The top quark mass is a basic input parameter for the Standard Model. Other precision tests of the Standard Model are compared to predictions that require an accurate value of the top quark mass. For example, an error of $600 \mathrm{MeV}$ in the top quark mass corresponds to an error of $5 \mathrm{MeV}$ for the prediction of the mass of the $W$ boson. At the ILC, we expect to measure the mass of the $W$ boson to a few $\mathrm{MeV}$, a level that gives sensitivity to loop corrections from a variety of predicted new particles [37].

A precise knowledge of the top quark mass is also relevant to an unusual prediction of the Standard Model. If there are no new particles below $10^{16} \mathrm{GeV}$, the Standard Model predicts that the potential for the Higgs field turns over at large values and eventually becomes negative. Then our universe is unstable over long time scales with respect to tunnelling to a ground state in which the Higgs field takes an extremely large vacuum value. The instability is driven by the interaction between the Higgs field and the top quark. The instability occurs if the top quark mass is greater than $171.1 \mathrm{GeV}$, a value about $2 \sigma$ below the currently measured value [38]. So, even if the Standard Model were literally correct, we would need to improve the measurement of the top quark to be confident of the ultimate fate of the universe.

\subsection{Top quark weak and electromagnetic couplings}

At higher energies, top quark and antiquark pairs are produced in the continuum through $s$-channel $\gamma$ and $Z$. The contributions from the $\gamma$ and $Z$ diagrams have $\mathcal{O}(1)$ interference, which is constructive or destructive depending on the electron and positron beam polarizations and the top quark polarizations. This generates $\mathcal{O}(1)$ forward-backward and polarization asymmetries, shown in Fig. 8, and also parityviolating asymmetries in the top quark decays. The top and antitop decays through weak interactions serve as polarization analyzers.

The central feature of the weak interaction, the feature that distinguishes it from the strong and electromagnetic interactions, and the feature that required the intervention of the Higgs field, is that the couplings depend on polarization. Making use of the unique capability of the ILC for polarized electron and positron beams, we will be able to measure the individual couplings of each polarization state of the top quark to the weak interaction bosons $W$ and $Z$. The measurement accuracies from the ILC should improve by about an order of magnitude over what is projected for the LHC. The discrimination of the left- and right-handed couplings to the $Z$ boson is a unique feature of the ILC measurements. ILC can also unambiguously determine the signs of the two couplings. With the full ILC data set, the experiments should 


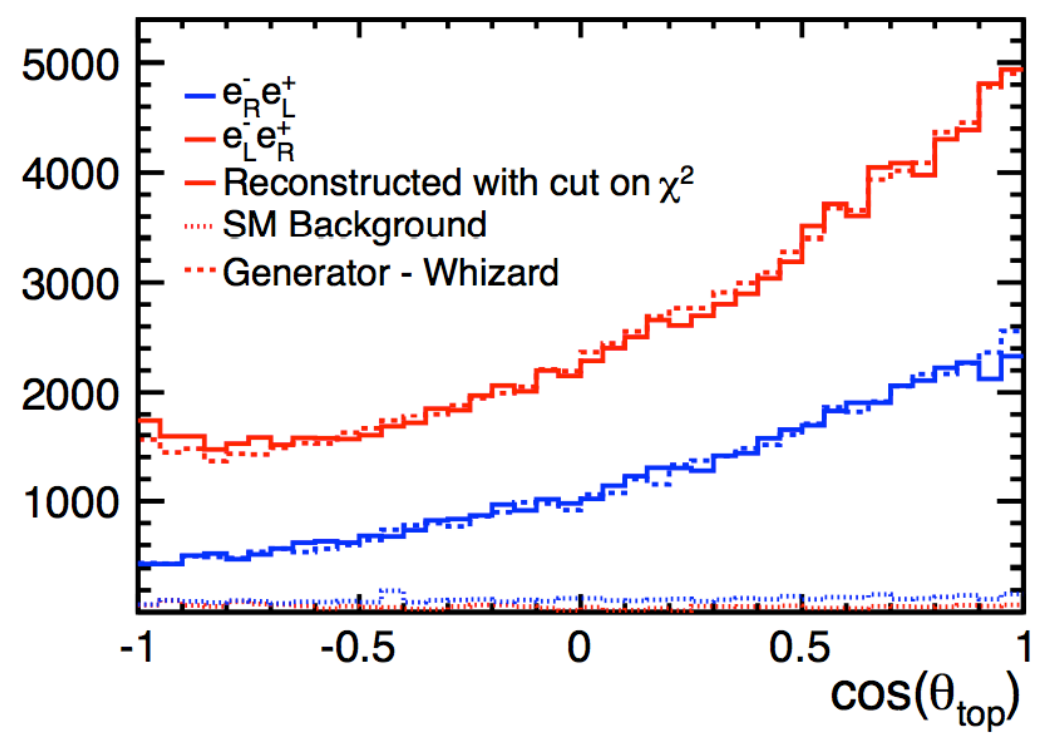

Figure 8: The angular distribution of produced top quarks obtained from full simulations based on a realistic detector model, full event reconstruction, and the inclusion of physics and machine-related backgrounds, compared to the corresponding generator-level distributions, from Ref. [40]

achieve a relative precision of $0.6 \%$ on the coupling of the left-handed top quark and $1.0 \%$ on the coupling of the right-handed top quark [39,40,41,42].

These polarization-dependent couplings receive corrections in most models of new physics beyond the Standard Model. The effects are particularly large in models in which the Higgs boson is a composite built of some more fundamental constituents. In such models, the shifts of the $t \bar{t} Z$ couplings can be $20 \%$ or larger and are expected to be different between the couplings to the two top quark polarization states. Figure 9 shows a survey of theoretical predictions collected in Ref. [41]. The separate values of these couplings provide a powerful diagnostic of the model. The measurement accuracies expected at the ILC and the LHC are also shown in the figure. Measurements with the ILC accuracy will not only establish the shifts of the $Z$ couplings with high significance but also pin down properties of the model that gave rise to them. A $1 \%$ measurement of these couplings is sensitive, in models of this type, to the presence of a 10-15 TeV Higgs-sector resonance coupling to $t \bar{t}$. This goes beyond the ultimate reach of the High-Luminosity LHC for direct searches for such a resonance, estimated to be about 5-6 TeV [36].

Full reconstruction of top quark pair events at the ILC will also allow us to search for exotic decay modes of the top quark, and for nonzero magnetic and electric dipole moments. The latter measurements provide a unique and powerful probe of $\mathrm{CP}$ - 


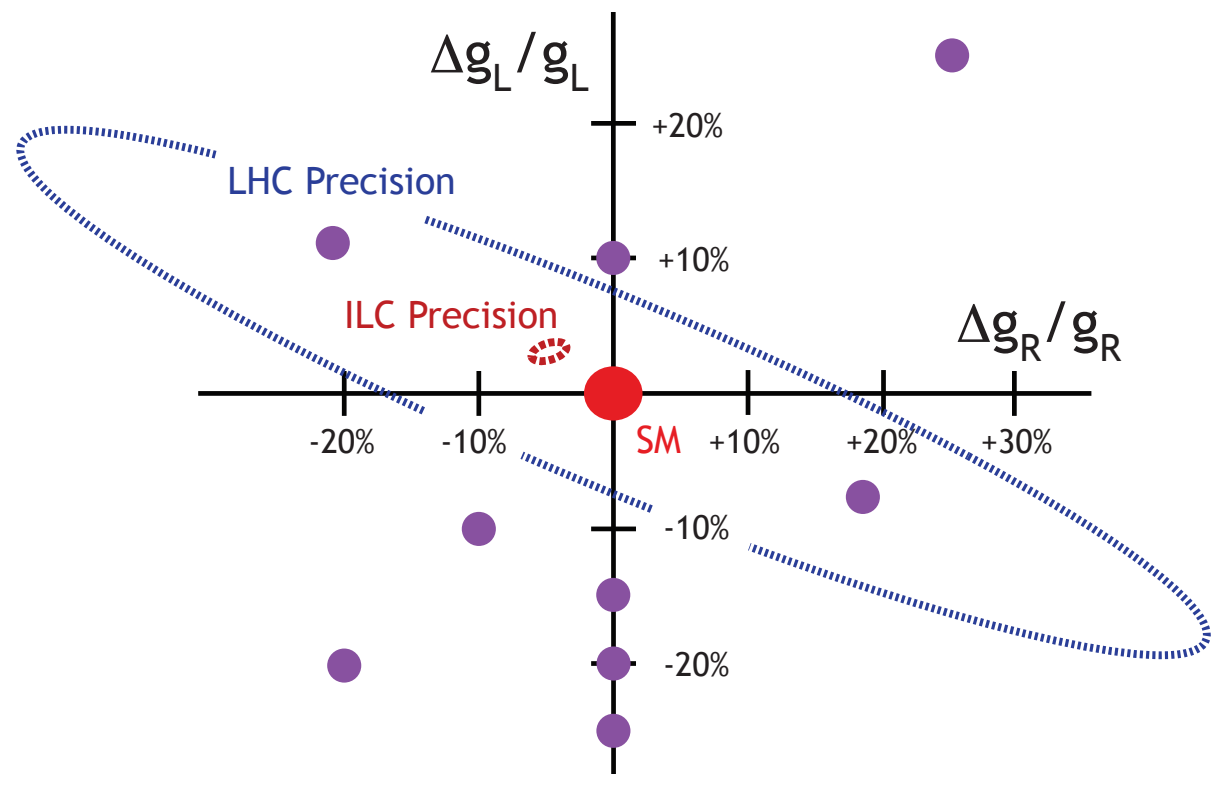

Figure 9: The heavy dots display the shifts in the left- and right-handed top quark couplings to the $Z$ boson predicted in a variety of models with composite Higgs bosons, from Ref. [41]. The ellipses show the $68 \%$ confidence regions for these couplings expected from the LHC [36,43] and the ILC [42].

violating interactions of the top quark $[44,45]$, which provide the driving force in one class of models of the cosmic matter-antimatter asymmetry.

\section{New Particles}

In addition to searches for new particles and forces through the precision study of the Higgs boson and the top quark, the ILC will carry out direct searches for new particles outside the Standard Model. The LHC has already carried out a broad program of searches for new particles, setting upper limits on masses higher than $1 \mathrm{TeV}$ in the best cases. Still, it is possible that new particles are being produced at the LHC and yet are not visible to the experiments there. Such particles do not appear only in artificial examples but even in some of the best-motivated scenarios for new physics. We will review some specific models of this type below. At the ILC, we can use the advantages of $e^{+} e^{-}$collisions to discover or definitively exclude these particles.

A new capability that the ILC will make available is the ability to polarize the colliding electron and positron beams. We have already discussed the use of beam polarization in studies of the Higgs boson and the top quark. For studies of an unknown 
new particle, this tool is even more powerful. By measuring the pair-production rate for the various beam polarizations, we can directly extract the quantum numbers of the particle under the electromagnetic and weak interactions. We will see illustrations of the power of beam polarization in the examples discussed below.

If a new particle is discovered or suggested, the energy of the ILC can be extended to reach the pair-production threshold. In addition, the ILC can schedule an energy scan near the pair production threshold, to obtain a very accurate measurement of the particle mass and quantum numbers.

There is a large literature on ILC searches for new particles, reviewed in Refs. [4,6]. In this section, we will review a few especially important examples. The specific examples will be taken from models with supersymmetry, the proposed fundamental symmetry linking fermions and bosons. However, the impressive capabilities of the ILC for new particle searches that these examples illustrate apply more generally. We will also review another precision probe for new physics, the precision study of two-fermion pair production.

\subsection{Hidden dark matter}

We have noted already that one of the most important questions in particle physics is the particle identity of the dark matter of the universe. Dark matter particles are difficult to observe at colliders. They are neutral and weakly interacting and thus make no signals in collider detectors. But if we are to understand dark matter, it will be extremely important to produce dark matter particles in a controlled environment, to measure their quantum numbers and couplings. Only then can we produce a constrained, testable theory of dark matter production in the early universe [46].

The most powerful strategy for studying dark matter at the LHC is to produce heavy, strongly interacting particles that decay to dark matter particles. The dark matter particles would be invisible to the LHC detectors, but the visible decay products that accompany them would give clues to their properties. Unfortunately, these heavier precursor particles have not yet been discovered, and they might in fact be too heavy to produce at the LHC.

In many models of dark matter, there is an electrically charged particle that can decay to the neutral dark matter particle. If the charged and neutral particles are well separated in mass, the visible particles emitted in the decay can be observed at the LHC. Using this strategy, the LHC experiments have excluded supersymmetric partners of the $W$ and $Z$ bosons with masses as high as $700 \mathrm{GeV}$ in the easiest cases $[47,48]$. However, many interesting cases are much more difficult for the LHC experiments. In particular, it is often true that the lightest neutral particle in these models has a small annihilation cross section, leading to too many dark matter particles surviving in the universe today. To obtain the observed amount of dark matter, 
the model should have "coannihilation", the simultaneous annihilation of charged and neutral states. This requires that the dark matter particle and its charged partner have a mass difference of $20 \mathrm{GeV}$ or less. This gives an example of what is called a "compressed" spectrum in the LHC literature. The emitted visible decay products are too soft to pass the triggers of the LHC detectors, and the process of particle production and decay, which may occur at a high rate, becomes unobservable.

A second strategy is to observe reactions with an initial state radiation gluon recoiling against invisible particles. This method allows discovery of the pair-production of invisible particles. Most studies for LHC have assumed that dark matter particles are produced by a pointlike contact interaction. The pointlike coupling leads to gluon radiation with large transverse momentum that can be used as a signature [49]. However, for models, including supersymmetric models, in which dark matter is produced more conventionally by electroweak interactions, this method becomes more difficult. The spectrum of initial state radiation is similar to that in the Drell-Yan process. There is a large background from production of a $Z$ boson which then decays to neutrinos, and from $W$ production with a final-state lepton unobserved. The systematic error from subtracting this background dominates the measurement. The result is that dark matter particles can only be discovered for masses of $100-200 \mathrm{GeV}$, depending on the electroweak quantum numbers, even with the High-Luminosity LHC [50]. A discovery would indicate that a new, invisible particle was produced but would tell us little about its properties.

At the ILC, we can search for production of invisible particles with initial state radiation in a way that addresses these issues. Because there is no large strong interaction background, the ILC experiments need no triggers and can detect emitted particles with energies below $1 \mathrm{GeV}$. Initial-state radiation is present as photons rather than gluons, so the background rates are much smaller and also precisely calculable. The background depends in a known way on beam polarization; this effect can be used to minimize the background and also to measure its influence. The sensitivity of the search for dark matter at the ILC through a search that only relies on initial state radiation has been studied in Ref. [51]. Any type of dark matter that annihilates to $e^{+} e^{-}$can be discovered with this technique (for high enough collider energy), even if this annihilation channel is only a few percent of the total annihilation rate.

The ILC experiments expect not only to observe initial state radiation but also to resolve soft particles produced in decays. For example, Ref. [52] considers a model in which the dark matter particle is the lightest supersymmetry partner $\chi$. The model is arranged so that the supersymmetry partner $\widetilde{\tau}$ of the tau lepton coannihilates with the dark matter particle $\chi$ to give the observed value of the cosmic dark matter density. In the model, the mass of the $\widetilde{\tau}$ is $107 \mathrm{GeV}$. The mass difference between the $\widetilde{\tau}$ and the $\chi$ is set by the coannihilation rate to be $11 \mathrm{GeV}$. Figure 10 shows an ILC simulation of pair-production of the supersymmetric partner $(\widetilde{\tau})$ of the tau lepton. The $\widetilde{\tau}$ decays to a $\chi$ and a soft tau lepton, which is observed in its decay to low-energy $\pi$ mesons. 


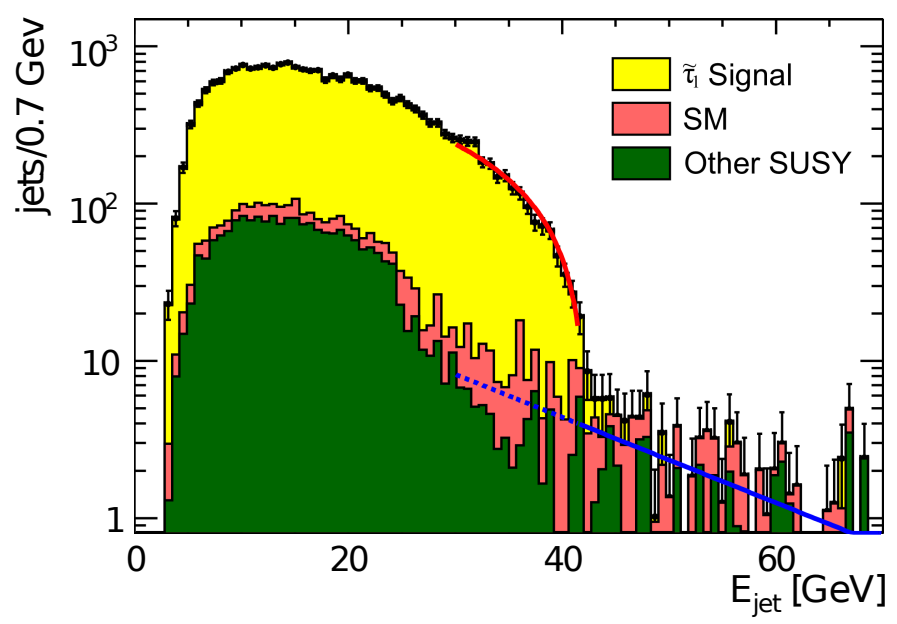

Figure 10: Energy distribution of hadronic decay products of the tau lepton in events selected for $\widetilde{\tau}^{+} \widetilde{\tau}^{-}$pair production at the ILC, from Ref. [52].

The $\widetilde{\tau}$ pair production signal, shown in yellow in the figure, stands out clearly above the various backgrounds. In the analysis of this model, the masses of the $\widetilde{\tau}$ and $\chi$ are determined to a precision of $200 \mathrm{MeV}$ and $400 \mathrm{MeV}$, respectively. The electroweak quantum numbers of the $\widetilde{\tau}$ are determined from the production rates with polarized beams. By combining this information with other information available from ILC measurements in this model, the annihilation rate of the $\chi$ can be determined and the cosmic density of $\chi$ dark matter can be predicted to $0.2 \%$ accuracy.

\subsection{Hidden Higgsino}

Supersymmetry is an especially attractive principle to extend the Standard Model. It gives a framework for the unification of the coupling constants of the strong, weak, and electromagnetic interactions and a raison d'être for the appearance of fundamental scalar fields such as the Higgs field. New particles predicted by supersymmetry have been searched for intensively at the LHC, though none have been found so far. Supersymmetry at the weak interaction scale is often motivated by its possible role in explaining the form of the Higgs potential. For this, some supersymmetric particles must have masses near the weak interaction mass scale. The strongest arguments can be made that the supersymmetric partners of the Higgs boson, called Higgsinos, should be relatively light and accessible to collider experiments [53].

However, the Higgsinos are especially difficult to discover at the LHC. They have all of the problems described for dark matter in the previous section. They are produced only by electroweak interactions. Though there must be both charged and neutral Higgsinos, supersymmetry predicts that their masses are naturally compressed 

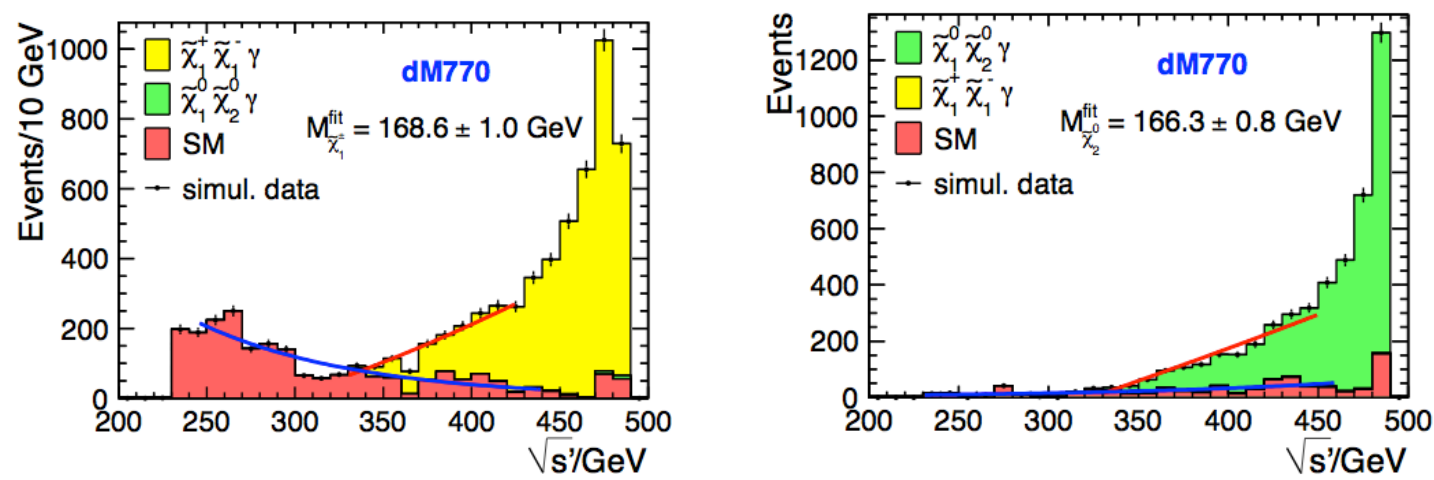

Figure 11: Distribution of the missing mass for a system recoiling against an initial state radiation photon in a model with Higgsino production at the ILC, from Ref. [54]. Left: events selected for charged Higgsinos. Right: events selected for neutral Higgsinos.

if the supersymmetric partners of the weak interaction gauge bosons are heavy. Thus it is not surprising that Higgsinos are hardly constrained by LHC data.

The observation of Higgsinos at the ILC has been studied in Ref. [54]. The more difficult model considered in this paper contains Higgsinos with masses near $165 \mathrm{GeV}$, with a mass difference of about $1 \mathrm{GeV}$ between the charged and the lighter neutral Higgsino. Nevertheless the signal of Higgsino production from initial state radiation photons is substantial, as shown in Fig. 11. The various Higgsino masses are determined to a precision of about $1 \mathrm{GeV}$. In addition, it is possible to observe soft $\pi$ mesons from the decay of the charged Higgsino, providing a very sharp determination of the mass difference between the Higgsino states. The mass differences provide an estimate of the masses of the supersymmetric partners of the electroweak gauge bosons, which are set to several $\mathrm{TeV}$ in this model.

From the rates for Higgsino production with polarized beams, we will be able to determine the quantum numbers of these particles. In this case, the cross section measurements can confirm that the particles discovered indeed have the quantum numbers expected for a Higgsino.

\subsection{Hidden pseudoscalar Higgs bosons}

Another context in which the ILC can make important additions to the LHC searches for new particle is in the search for additional Higgs bosons. We have already discussed the prospects for searching for such bosons indirectly through shifts in couplings of the known Higgs bosons to quarks and leptons. There are also many scenarios in which additional Higgs bosons are relatively light but difficult to discover at the LHC. For example, a model with two Higgs fields contains a Higgs boson of 
odd parity that decays primarily to heavy quarks and leptons. This boson can be as light as the known Higgs boson, or even lighter, due to additional global symmetries of the Higgs sector. Because of its parity, its coupling to the $W$ and $Z$ bosons is suppressed. If the mass of this particle were below $10 \mathrm{GeV}$, it would be seen in $\Upsilon$ decays; above $200 \mathrm{GeV}$, it could be seen at the LHC in decays to two photons or two gluons. Between these limits, the LHC must search for these particles using electroweak production and purely leptonic decays, a difficult prospect even for the high-luminosity era. At the ILC running at $500 \mathrm{GeV}$, this Higgs boson is produced by radiation from top or bottom quarks. It can be discovered straightforwardly in its dominant $b \bar{b}$ and $\tau^{+} \tau^{-}$decay modes [55]. If the mass of the odd parity Higgs boson is less than $62 \mathrm{GeV}$, it can also be discovered as an exotic decay product of the known Higgs boson [56].

\subsection{Two-fermion processes}

The ILC will also be able to search for new gauge bosons, making use of its capability for precision measurement of the basic two-fermion processes $e^{+} e^{-} \rightarrow \ell^{+} \ell^{-}$ and $e^{+} e^{-} \rightarrow q \bar{q}$. A new neutral gauge boson $Z^{\prime}$ will perturb the cross sections predicted in the Standard Model through interference with the production diagrams involving the $\gamma$ and $Z$. The Standard Model expectation is understood theoretically at the $0.1 \%$ level, so these measurements are sensitive to $Z^{\prime}$ bosons well above the collider center of mass energy.

New $Z^{\prime}$ bosons appear, for example, in models of the grand unification of the strong, weak, and electromagnetic interactions. The gauge group that unifies the known interactions may include other, new, interactions as well. Figure 12 shows an analysis of a model with a $Z^{\prime}$ boson with the quantum numbers expected from the grand unification group $S O(10)$. In the example studied, the boson is assumed to have a mass of $3 \mathrm{TeV}$. Such a boson has been searched for at the LHC as a resonance in lepton pair production and excluded for masses up to $2.6 \mathrm{TeV}$ [57]. If the resonance is present at a higher value of the mass, the figure illustrates that the ILC will give significant information about its pattern of couplings. If the resonance is not present, the ILC will put a lower bound on its mass at $7 \mathrm{TeV}$, comparable to the projected LHC bound of $6 \mathrm{TeV}$. Running of the ILC at $1000 \mathrm{GeV}$ would roughly double its search reach [1].

If a $Z^{\prime}$ is indeed present in the region that will be explored by the LHC and the ILC, there will be impressive synergy between the measurements at the two colliders. The LHC experiments will observe the $Z^{\prime}$ directly as a resonance and measure its mass accurately. The ILC will then measure the couplings of the $Z^{\prime}$ to each individual quark and lepton species, taking advantage of beam polarization to measure both the left-handed and right-handed couplings in each case. With this information, one 

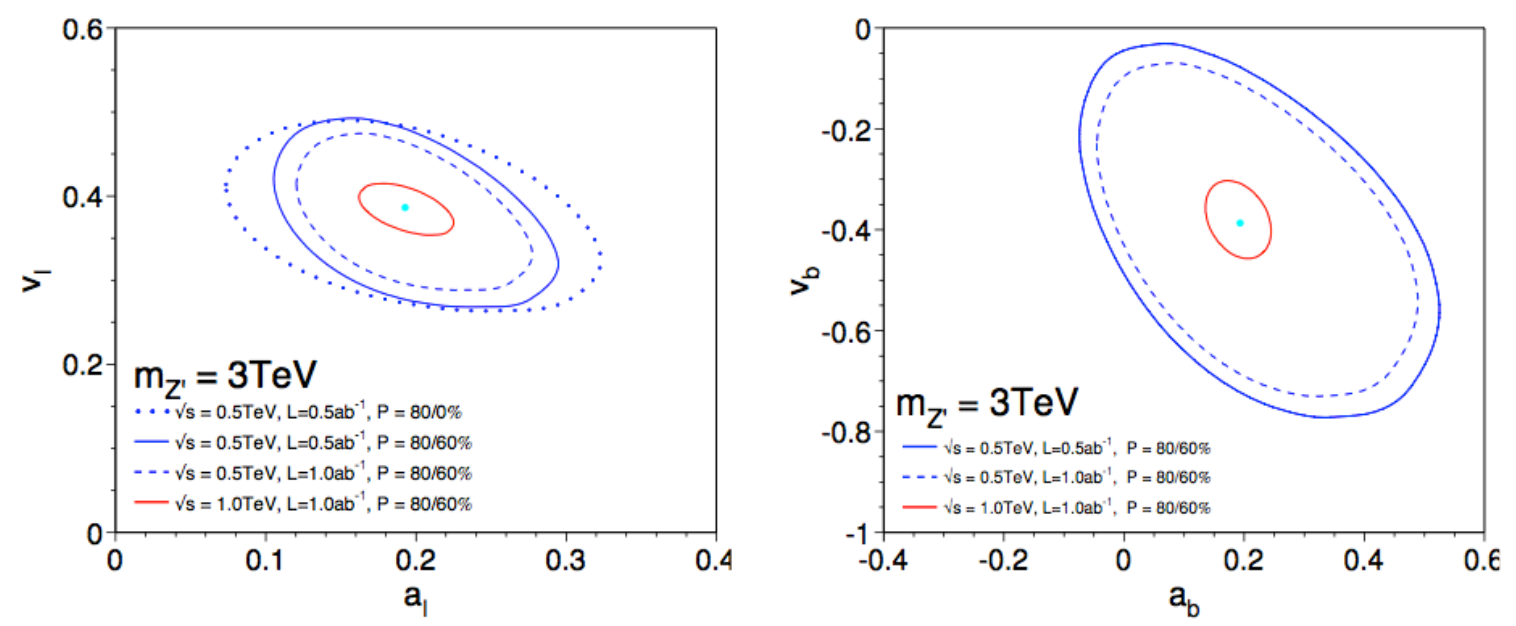

Figure 12: Determination of the vector and axial couplings of a $Z^{\prime}$ resonance to leptons (left panel) and $b$ quarks (right panel) through measurement of $e^{+} e^{-} \rightarrow \ell^{+} \ell^{-}$and $e^{+} e^{-} \rightarrow b \bar{b}$ at the ILC, from Ref. [1].

can fully identify the gauge boson and find its place in an extended gauge theory of nature.

Other effects can also perturb the two-fermion processes. If quarks and leptons are composite, the first manifestation of this will be the perturbation of two-fermion processes by the effects of higher-dimension operators. This effect can be searched for at the LHC, but it requires careful calibration of quark jet signals at the highest energies. In addition, limits from the LHC are model-dependent because many operators can potentially contribute. For this reason, the best current constraints on quark and lepton compositeness still come from the data from the $e^{+} e^{-}$collider LEP, putting limits on the compositeness scale at about $10 \mathrm{TeV}$. The ILC, with higher energy, luminosity, and intrinsic precision and also the capability for electron and positron beam polarization, should improve these constraints by an order of magnitude in the compositeness scale [1].

\section{Conclusion}

In this report, we have surveyed the major elements of the ILC physics program. We have reviewed the ILC capabilities to search for new particles and interactions through precision studies of the Higgs boson and the top quark, and we have reviewed the capabilities of the ILC to carry out direct searches for possible new particles.

The discovery of the Higgs boson at the LHC has completed the construction 
of a Standard Model for particle physics. That model is potentially complete and internally consistent. Howver, it is also inadequate to answer the many open questions that remain in particle physics. If these questions have answers, the Standard Model must break down. Researchers in our community are asking where and how that breakdown can be discovered.

The ILC offers new avenues to address that question experimentally. It offers new, precise, unambigous information on the two elementary particles most closely connected to our questions about the Standard Model, the top quark and the Higgs boson itself.

Though the LHC has carried out many searches for new particles, there are gaps in those searches reflecting the difficulty of experimentation at hadron colliders. The ILC will bring new capabilities that will allow crucial searches for a variety of wellmotived new physics scenarios such as supersymmetry, Higgs compositeness, new gauge bosons, additional Higgs bosons, and particles connected with cosmic dark matter that may not be possible to see by any other means.

From these arguments, we know today that the ILC has the potential to make major contributions to particle physics. As the LHC winds down its program fifteen years from now, the ILC will become the world's most important source of new information on the issues that surround the Standard Model. If new physics beyond the Standard Model is discovered in that interval, through results from the LHC, from dark matter detection, from cosmology, or from other sources, so much the better.

Finally, we note that, although the estimates of performance of the ILC experiments given in this report are done as realistically as possible at this stage, it is another question to extract these levels of performance from a running experiment. The ATLAS and CMS collaborations have now met and even exceeded the performance they projected before the startup of the LHC. But this accomplishment took the hard work of many people, in optimizing and then constructing the detectors, understanding the actual environment provided by the accelerator, producing precise calculations and simulations, and collecting and analyzing the data in this context. For the ILC, a similar effort will be needed to carry out the program we have outlined here. We hope that readers of this paper will join us in this endeavor to realize the promise offered by the ILC.

\section{ACKNOWLEDGEMENTS}

We thank James Brau, Roberto Contino, and Junping Tian for their assistance in preparing this report. We are grateful to many other members of the Linear Collider community for discussions of the issues reviewed here. 


\section{A Appendix: Table of ILC projected uncertainties}

In this appendix, we list the current projections from the ILC detector groups for the expected accuracy with which the most important physics parameters constrained by the ILC will be measured. We recommend that these numbers be used in discussions of the ILC physics prospects and in comparisons of the ILC with other proposed facilities.

Projected accuracies for the ILC depend on the run plan assumed. Following the report of the ILC Parameters Joint Working Group [7], we assume the following scenario: In its initial phase, the ILC would accumulate $500 \mathrm{fb}^{-1}$ at $500 \mathrm{GeV}$, then $200 \mathrm{fb}^{-1}$ at $350 \mathrm{GeV}$, then $500 \mathrm{fb}^{-1}$ at $250 \mathrm{GeV}$. After a luminosity upgrade, the ILC would accumulate an additional $3500 \mathrm{fb}^{-1}$ at $500 \mathrm{GeV}$, then $1500 \mathrm{fb}^{-1}$ at $250 \mathrm{GeV}$. Based on the accelerator design described in the ILC TDR [58], this program would require 8.1 calendar years, including a realistic start-up profile, to complete the initial phase. It would require a total of 20.2 calendar years to complete the whole program, including the downtime needed for the upgrade. The full ILC data set would then include $2 \mathrm{ab}^{-1}$ at $250 \mathrm{GeV}, 200 \mathrm{fb}^{-1}$ at $350 \mathrm{GeV}$ and $4 \mathrm{ab}^{-1}$ at $500 \mathrm{GeV}$. More details and some alternative scenarios are given in [7].

Table 1 gives the corresponding projections for the uncertainties in physics parameters. The numbers listed are obtained from full-simulation analyses using the ILD and SiD detector models described in Ref. [59]. These are Geant4-based simulations with detailed detector designs, which have in many cases been confirmed by test beam measurements on detector prototypes. For each number, we have given a reference in which the method is described. The actual number given may reflect more recent improvements in the analysis [60]. The uncertainties include both expected statistical and systematic errors.

The estimated uncertainties for the Higgs boson couplings are based on a fit to ILC observables in which all individual couplings (including the loop-induced couplings to $g g$ and $\gamma \gamma$ ) are varied independently. The total width of the Higgs boson is also taken as an independent variable, to account for exotic Higgs decays not constrained by any direct measurement. (Higgs decay to invisible states is directly observed using the $h Z$ production process.) The constraints on the Higgs boson derived from this analysis are completely model-independent.

The second line for $g(h \gamma \gamma)$ assumes that the ILC data are combined with an LHC measurement of the ratio of branching ratios $\Gamma(h \rightarrow \gamma \gamma) / \Gamma\left(h \rightarrow Z Z^{*}\right)$ to $2 \%$ accuracy, as projected by the ATLAS collaboration for the High-Luminosity LHC [16]. This is the only place where combination with projected LHC results significantly improves the model-independent ILC results.

For comparison with results from hadron colliders, where a model-independent 
analysis is not possible, the 2013 Snowmass study [18] suggested a fit to observables in which one adds the model assumptions that $g(h c \bar{c}) / g(h t \bar{t})$ and $g(h \mu \mu) / g(h \tau \tau)$ have their Standard Model values and that the Higgs boson has no invisible or exotic decays. The results of that analysis are given in Table 2 .

Results from the analysis of measurements of the top quark threshold are dominated by theoretical systematic errors from the theoretcial calculation of the threshold cross section shape. These errors are estimated conservatively based on a new calculation of this cross section at the $\mathrm{N}^{3} \mathrm{LO}$ level [31,32]. Measurements at any $e^{+} e^{-}$ collider should show these same uncertainties. The statistical errors from the ILC program are quoted in the main text.

The estimated uncertainties for the top quark electroweak couplings are analyzed in the following way [42]: First a fit is done with the four chirality-conserving couplings $g_{L}^{\gamma}, g_{R}^{\gamma}, g_{L}^{Z}, g_{R}^{Z}$ taken to be independent parameters and the chirality flip couplings taken to be zero. Then a fit is done with the chirality conserving parameters taken at their Standard Model values and the two CP-conserving chirality-flip parameters

$F_{2}^{\gamma}, F_{2}^{Z}$ taken as independent free parameters. For the high-luminosity estimates, we have conservatively added a $0.5 \%$ systematic uncertainty.

The limits on dark matter production are based on an effective field theory analysis as described in Ref. [51]. Dark matter pair production is represented by a contact interaction with the scale $\Lambda$; the labels D5 and D8 refer to two possible spin structures. We emphasize that the effective field theory approximation is accurate in this analysis, while it is questionable in similar analyses for hadron colliders. The quoted limits are based on a full-simulation study described in Ref. [61].

\section{References}

[1] H. Baer, et al., "The International Linear Collider Technical Design Report - Volume 2: Physics," http://www.linearcollider.org/ILC/Publications/ Technical-Design-Report, arXiv:1306.6352 [hep-ph].

[2] D. M. Asner, et al., "ILC Higgs White Paper," in the Proceedings of the APS DPF Community Summer Study (Snowmass 2013), arXiv:1310.0763 [hep-ph].

[3] D. Asner, et al., "Top quark precision physics at the International Linear Collider," in the Proceedings of the APS DPF Community Summer Study (Snowmass 2013), arXiv:1307.8265 [hep-ex].

[4] H. Baer, et al., "Physics Case for the ILC Project: Perspective from Beyond the Standard Model," in the Proceedings of the APS DPF Community Summer Study (Snowmass 2013), arXiv:1307.5248 [hep-ph]. 
[5] A. Freitas, et al. "Exploring Quantum Physics at the ILC," in the Proceedings of the APS DPF Community Summer Study (Snowmass 2013), arXiv:1307.3962 [hep-ph].

[6] G. Moortgat-Pick, et al., "Physics at the $e^{+} e^{-}$Linear Collider," arXiv:1504.01726 [hep-ph], to appear in EPJC.

[7] T. Barklow, et al. [ILC Parameters Joint Working Group], "ILC Operating Scenarios", ILC-NOTE-2015-068, arXiv:1506.07830.

[8] ATLAS Collaboration, Phys. Lett. B 716 (2012) 1, arXiv:1207.7214 [hep-ex].

[9] CMS Collaboration, Phys. Lett. B 716 (2012) 30, arXiv:1207.7235 [hep-ex].

[10] C. Adolphsen, et al., http://www.linearcollider.org/ILC/Publications/ Technical-Design-Report, arXiv:1306.6328 [physics.acc-ph].

[11] V. Khachatryan et al. [CMS Collaboration], Eur. Phys. J. C 75, no. 5, 212 (2015) [arXiv:1412.8662 [hep-ex]].

[12] I. Low, R. Rattazzi and A. Vichi, JHEP 1004, 126 (2010) [arXiv:0907.5413 [hep-ph]].

[13] M. Farina, M. Perelstein and N. R. L. Lorier, Phys. Rev. D 90, 015014 (2014) [arXiv:1305.6068 [hep-ph]].

[14] The events from $Z$ fusion can also contribute significantly to the measurement of the $h Z Z$ couplings; see T. Han, Z. Liu, Z. Qian and J. Sayre, arXiv:1504.01399 [hep-ph].

[15] H. Li et al. [ILD Design Study Group Collaboration], arXiv:1202.1439 [hep-ex]; S. Watanuki, in preparation.

[16] ATLAS Collaboration, ATL-PHY-PUB-2014-016 (2014).

[17] M. E. Peskin, in the Proceedings of the APS DPF Community Summer Study (Snowmass 2013), arXiv:1312.4974 [hep-ph].

[18] S. Dawson, et al., in the Proceedings of the APS DPF Community Summer Study (Snowmass 2013), arXiv:1310.8361 [hep-ex].

[19] CMS Collaboration, in the Proceedings of the APS DPF Community Summer Study (Snowmass 2013), arXiv:1307.7135.

[20] S. Kanemura, K. Tsumura, K. Yagyu and H. Yokoya, Phys.Rev. D90, 075001 (2014) [arXiv:1406.3294 [hep-ph]]. 
[21] K. Kajantie, M. Laine, K. Rummukainen and M. E. Shaposhnikov, Phys. Rev. Lett. 77, 2887 (1996) [hep-ph/9605288].

[22] C. Grojean, G. Servant and J. D. Wells, Phys. Rev. D 71, 036001 (2005) [hepph/0407019], A. Noble and M. Perelstein, Phys. Rev. D 78, 063518 (2008) [arXiv:0711.3018 [hep-ph]].

[23] For example, M. Aoki, S. Kanemura and O. Seto, Phys. Rev. Lett. 102, 051805 (2009) [arXiv:0807.0361 [hep-ph]].

[24] ATLAS Collaboration, ATL-PHY-PUB-2014-019 (2014).

[25] J. Tian, LC-REP-2013-003, http://www-flc.desy.de/lcnotes/notes/ LC-REP-2013-003.pdf.

[26] M. Kurata et al, LC-REP-2013-025, http://www-flc.desy.de/lcnotes/ notes/LC-REP-2013-025.pdf.

[27] J. Walsh [for the BaBar and Belle Collaborations], J. Phys. Conf. Ser. 447, 012013 (2013).

[28] A. J. Bevan et al. [BaBar and Belle Collaborations], "The Physics of the B Factories," arXiv:1406.6311 [hep-ex].

[29] A. H. Hoang, et al., Eur. Phys. J. direct C 2, 1 (2000) [hep-ph/0001286], A. H. Hoang and M. Stahlhofen, JHEP 1405, 121 (2014) [arXiv:1309.6323 [hep$\mathrm{ph}]$.

[30] F. Bach and M. Stahlhofen, arXiv:1411.7318 [hep-ph]; F. Bach, B. Chokoufe, A. Hoang, J. Reuter, M. Stahlhofen, and C. Weiss, in preparation.

[31] M. Beneke and M. Steinhauser, Nucl. Part. Phys. Proc. 261-262, 378 (2015); M. Beneke, Y. Kiyo, P. Marquard, J. Piclum, A. Penin and M. Steinhauser, arXiv:1506.06864 [hep-ph].

[32] M. Beneke, A. Maier, J. Piclum and T. Rauh, arXiv:1506.06865 [hep-ph].

[33] K. Seidel, F. Simon, M. Tesar and S. Poss, Eur. Phys. J. C 73, 2530 (2013).

[34] T. Horiguchi, A. Ishikawa, T. Suehara, K. Fujii, Y. Sumino, Y. Kiyo and H. Yamamoto, arXiv:1310.0563 [hep-ex].

[35] P. Marquard, A. V. Smirnov, V. A. Smirnov and M. Steinhauser, Phys. Rev. Lett. 114, no. 14, 142002 (2015) [arXiv:1502.01030 [hep-ph]].

[36] K. Agashe et al. "Working Group Report: Top Quark," in the Snowmass 2013 proceedings, arXiv:1311.2028 [hep-ph]. 
[37] M. Baak, et al., "Working Group Report: Precision Study of Electroweak Interactions," in the Snowmass 2013 proceedings, arXiv:1310.6708 [hep-ph].

[38] G. Degrassi et al., JHEP 1208, 098 (2012) [arXiv:1205.6497 [hep-ph]]; D. Buttazzo, et al., JHEP 1312, 089 (2013) [arXiv:1307.3536 [hep-ph]].

[39] E. Devetak, A. Nomerotski and M. Peskin, Phys. Rev. D 84, 034029 (2011) [arXiv:1005.1756 [hep-ex]].

[40] M. S. Amjad, et al., arXiv:1307.8102 [hep-ex].

[41] F. Richard, arXiv:1403.2893 [hep-ph]. The details of the theoretical predictions shown in Fig. 8, and the references to the literature, can be found there.

[42] M. S. Amjad, et al., arXiv:1505.06020 [hep-ex].

[43] R. Röntsch and M. Schulze, JHEP 1407, 091 (2014) [arXiv:1404.1005 [hep-ph]], arXiv:1501.05939 [hep-ph].

[44] J. A. Aguilar-Saavedra et al. [ECFA/DESY LC Physics Working Group Collaboration], hep-ph/0106315.

[45] B. Grzadkowski and Z. Hioki, Nucl. Phys. B 585, 3 (2000) [hep-ph/0004223].

[46] E. A. Baltz, M. Battaglia, M. E. Peskin and T. Wizansky, Phys. Rev. D 74, 103521 (2006) [hep-ph/0602187].

[47] G. Aad et al. [ATLAS Collaboration], JHEP 1405, 071 (2014) [arXiv:1403.5294 [hep-ex]].

[48] V. Khachatryan et al. [CMS Collaboration], arXiv:1405.7570 [hep-ex].

[49] N. Zhou, D. Berge and D. Whiteson, Phys. Rev. D 87, 095013 (2013) [arXiv:1302.3619 [hep-ex]].

[50] M. Low and L. T. Wang, JHEP 1408, 161 (2014) [arXiv:1404.0682 [hep-ph]].

[51] Y. J. Chae and M. Perelstein, JHEP 1305, 138 (2013) [arXiv:1211.4008 [hep-ph]].

[52] M. Berggren, A. Cakir, D. Krücker, J. List, A. Lobanov and I. A. MelzerPellmann, arXiv:1307.8076 [hep-ph].

[53] H. Baer, V. Barger, P. Huang, D. Mickelson, A. Mustafayev and X. Tata, in the Proceedings of the APS DPF Community Summer Study (Snowmass 2013), arXiv:1306.2926 [hep-ph].

[54] M. Berggren, F. Brümmer, J. List, G. Moortgat-Pick, T. Robens, K. Rolbiecki and H. Sert, Eur. Phys. J. C 73, 2660 (2013) [arXiv:1307.3566 [hep-ph]]. 
[55] W. Kilian, D. Rainwater and J. Reuter, Phys. Rev. D 71, 015008 (2005) [hep$\mathrm{ph} / 0411213]$.

[56] T. Liu and C. T. Potter, in the Proceedings of the APS DPF Community Summer Study (Snowmass 2013), arXiv:1309.0021 [hep-ph].

[57] G. Aad et al. [ATLAS Collaboration], arXiv:1405.4123 [hep-ex].

[58] C. Adophsen, et al., "The International Linear Collider Technical Design Report - Volume 3: Accelerator," http://www.linearcollider.org/ILC/ Publications/Technical-Design-Report, arXiv:1306.6328, arXiv1306.6353 [physics.acc-ph].

[59] T. Behnke, et al., "The International Linear Collider Technical Design Report Volume 4: Detectors," http://www.linearcollider.org/ILC/Publications/ Technical-Design-Report, arXiv:1306.6329 [physics.ins-det].

[60] More information about the updated analyses can be found in Ref. [7] and in workshop talks cited there.

[61] C. Bartels, M. Berggren and J. List, Eur. Phys. J. C 72, 2213 (2012) [arXiv:1206.6639 [hep-ex]];

[62] M. Baak, et al., in the Proceedings of the APS DPF Community Summer Study (Snowmass 2013), arXiv:1310.6708 [hep-ph].

[63] J. List [ILD and SiD Collaborations], PoS EPS -HEP2013, 233 (2013). 


\begin{tabular}{|c|c|c|c|c|c|}
\hline Topic & Parameter & Initial Phase & Full Data Set & units & ref. \\
\hline \multirow[t]{15}{*}{ Higgs } & $m_{h}$ & 25 & 15 & $\mathrm{MeV}$ & {$[15]$} \\
\hline & $g(h Z Z)$ & 0.58 & 0.31 & $\%$ & {$[2]$} \\
\hline & $g(h W W)$ & 0.81 & 0.42 & $\%$ & [2] \\
\hline & $g(h b \bar{b})$ & 1.5 & 0.7 & $\%$ & [2] \\
\hline & $g(h g g)$ & 2.3 & 1.0 & $\%$ & [2] \\
\hline & $g(h \gamma \gamma)$ & 7.8 & 3.4 & $\%$ & {$[2]$} \\
\hline & & 1.2 & 1.0 & $\%, \mathrm{w} . \mathrm{LHC}$ results & {$[17]$} \\
\hline & $g(h \tau \tau)$ & 1.9 & 0.9 & $\%$ & [2] \\
\hline & $g(h c \bar{c})$ & 2.7 & 1.2 & $\%$ & [2] \\
\hline & $g(h t \bar{t})$ & 18 & 6.3 & $\%$, direct & [2] \\
\hline & & 20 & 20 & $\%, t \bar{t}$ threshold & {$[34]$} \\
\hline & $g(h \mu \mu)$ & 20 & 9.2 & $\%$ & {$[2]$} \\
\hline & $g(h h h)$ & 77 & 27 & $\%$ & {$[2]$} \\
\hline & $\Gamma_{t o t}$ & 3.8 & 1.8 & $\%$ & [2] \\
\hline & $\Gamma_{i n v i s}$ & 0.54 & 0.29 & $\%, 95 \%$ conf. limit & [2] \\
\hline \multirow[t]{8}{*}{ Top } & $m_{t}$ & 50 & 50 & $\operatorname{MeV}\left(m_{t}(1 \mathrm{~S})\right)$ & {$[33]$} \\
\hline & $\Gamma_{t}$ & 60 & 60 & $\mathrm{MeV}$ & {$[34]$} \\
\hline & $g_{L}^{\gamma}$ & 0.8 & 0.6 & $\%$ & {$[42]$} \\
\hline & $g_{R}^{\gamma}$ & 0.8 & 0.6 & $\%$ & {$[42]$} \\
\hline & $g_{L}^{Z}$ & 1.0 & 0.6 & $\%$ & {$[42]$} \\
\hline & $g_{R}^{Z}$ & 2.5 & 1.0 & $\%$ & {$[42]$} \\
\hline & $F_{2}^{\gamma}$ & 0.001 & 0.001 & absolute & {$[42]$} \\
\hline & $F_{2}^{Z}$ & 0.002 & 0.002 & absolute & {$[42]$} \\
\hline \multirow[t]{4}{*}{$W$} & $m_{W}$ & 2.8 & 2.4 & $\mathrm{MeV}$ & {$[62]$} \\
\hline & $g_{1}^{Z}$ & $8.5 \times 10^{-4}$ & $6 \times 10^{-4}$ & absolute & {$[63]$} \\
\hline & $\kappa_{\gamma}$ & $9.2 \times 10^{-4}$ & $7 \times 10^{-4}$ & absolute & {$[63]$} \\
\hline & $\lambda_{\gamma}$ & $7 \times 10^{-4}$ & $2.5 \times 10^{-4}$ & absolute & {$[63]$} \\
\hline \multirow[t]{2}{*}{ Dark Matter } & $\mathrm{EFT} \Lambda:$ D5 & 2.3 & 3.0 & TeV, 90\% conf. limit & {$[61]$} \\
\hline & $\operatorname{EFT} \Lambda:$ D8 & 2.2 & 2.8 & $\mathrm{TeV}, 90 \%$ conf. limit & {$[61]$} \\
\hline
\end{tabular}

Table 1: Projected accuracies of measurements of Standard Model parameters at the two stages of the ILC program proposed in the report of the ILC Parameters Joint Working Group [7]. This program has an initial phase with $500 \mathrm{fb}^{-1}$ at $500 \mathrm{GeV}, 200 \mathrm{fb}^{-1}$ at $350 \mathrm{GeV}$, and $500 \mathrm{fb}^{-1}$ at $250 \mathrm{GeV}$, and a luminosity-upgraded phase with an additional $3500 \mathrm{fb}^{-1}$ at $500 \mathrm{GeV}$ and $1500 \mathrm{fb}^{-1}$ at $250 \mathrm{GeV}$. Initial state polarizations are taken according to the prescriptions of [7]. Uncertainties are listed as $1 \sigma$ errors (except where indicated), computed cumulatively at each stage of the program. These estimated errors include both statistical uncertainties and theoretical and experimental systematic uncertainties. Except where indicated, errors in percent (\%) are fractional uncertainties relative to the Standard Model values. More specific information for the sets of measurements is given in the text. For each measurement, a reference describing the technique is given. 


\begin{tabular}{cc|c|c|c} 
Topic & Parameter & Initial Phase & Full Data Set & \\
\hline Higgs & $g(h Z Z)$ & 0.37 & 0.2 & $\%$ \\
& $g(h W W)$ & 0.51 & 0.24 & $\%$ \\
$g(h b \bar{b})$ & 1.1 & 0.49 & $\%$ \\
$g(h g g)$ & 2.1 & 0.95 & $\%$ \\
$g(h \gamma \gamma)$ & 7.7 & 3.4 & $\%$ \\
$g(h \tau \tau), g(\mu \mu)$ & 1.5 & 0.73 & $\%$ \\
$g(h c \bar{c}), g(h t \bar{t})$ & 2.5 & 1.1 & $\%$ \\
$\Gamma_{\text {tot }}$ & 1.8 & 0.96 & $\%$ \\
\hline
\end{tabular}

Table 2: Projected accuracies of measurements of Higgs boson couplings at the two stages of the ILC program, from the model-dependent fit used in the Snowmass 2013 study [18]. The analysis is as described in [2]. The ILC run plan assumed is the same as in Table 1. 